\title{
Increased Anxiety-Related Behavior, Impaired Cognitive Function and Cellular Alterations in the Brain of Cend1-deficient Mice
}

\author{
Katerina Segklia ${ }^{1 \dagger}$, Antonios Stamatakis ${ }^{2 \dagger}$, Fotini Stylianopoulou ${ }^{2}$, \\ Alexandros A. Lavdas ${ }^{1 \neq \S}$ and Rebecca Matsas ${ }^{1 * \neq}$ \\ ${ }^{1}$ Laboratory of Cellular and Molecular Neurobiology-Stem Cells, Department of Neurobiology, Hellenic Pasteur Institute, \\ Athens, Greece, ${ }^{2}$ Biology-Biochemistry Lab, Faculty of Nursing, School of Health Sciences, National and Kapodistrian \\ University of Athens, Athens, Greece
}

OPEN ACCESS

Edited by:

Rena Li,

The Roskamp Institute, United States

Reviewed by: Andras Bilkei-Gorzo, Universität Bonn, Germany Muddanna Sakkattu Rao, Kuwait University, Kuwait

*Correspondence: Rebecca Matsas rmatsa@pasteur.gr

${ }^{\dagger}$ These authors have contributed equally to this work

‡Equal senior authorship

$\S$ Present address: Alexandros A. Lavdas, Institute for Biomedicine, Eurac Research, Affiliated Institute of the University of Lübeck, Bolzano, Italy

Received: 30 July 2018 Accepted: 03 December 2018 Published: 29 January 2019

Citation: Segklia K, Stamatakis A, Stylianopoulou F, Lavdas AA and Matsas R (2019) Increased Anxiety-Related Behavior, Impaired

Cognitive Function and Cellular Alterations in the Brain of

Cend1-deficient Mice.

Front. Cell. Neurosci. 12:497. doi: 10.3389/fncel.2018.00497
Cend1 is a neuronal-lineage specific modulator involved in coordination of cell cycle exit and differentiation of neuronal precursors. We have previously shown that Cend1-/mice show altered cerebellar layering caused by increased proliferation of granule cell precursors, delayed radial granule cell migration and compromised Purkinje cell differentiation, leading to ataxic gait and deficits in motor coordination. To further characterize the effects of Cend1 genetic ablation we determined herein a range of behaviors, including anxiety and exploratory behavior in the elevated plus maze (EPM), associative learning in fear conditioning, and spatial learning and memory in the Morris water maze (MWM). We observed significant deficits in all tests, suggesting structural and/or functional alterations in brain regions such as the cortex, amygdala and the hippocampus. In agreement with these findings, immunohistochemistry revealed reduced numbers of $\gamma$ amino butyric acid (GABA) GABAergic interneurons, but not of glutamatergic projection neurons, in the adult cerebral cortex. Reduced GABAergic interneurons were also observed in the amygdala, most notably in the basolateral nucleus. The paucity in GABAergic interneurons in adult Cend1-/- mice correlated with increased proliferation and apoptosis as well as reduced migration of neuronal progenitors from the embryonic medial ganglionic eminence (MGE), the origin of these cells. Further we noted reduced GABAergic neurons and aberrant neurogenesis in the adult dentate gyrus (DG) of the hippocampus, which has been previously shown to confer spatial learning and memory deficits. Our data highlight the necessity of Cend1 expression in the formation of a structurally and functionally normal brain phenotype.

Keywords: GABAergic interneurons, neurogenesis, ganglionic eminence, somatosensory cortex, basolateral amygdala, dentate gyrus

Abbreviations: BLA, basolateral amygdala; CB, calbindin; DCX, doublecortin; DG, dentate gyrus; EPM, elevated plus maze; GABA, $\gamma$ - amino butyric acid; GE, ganglionic eminence; GFAP, glial fibrillary acidic protein; MGE, medial ganglionic eminence; MWM, Morris water maze; NDS, normal donkey serum; PBS, phosphate-buffered saline; PH3, phosphohistone 3; PV, parvalbumin; SGZ, subgranular zone; Sst, somatostatin; wt, wild type. 


\section{INTRODUCTION}

During brain development neural stem and progenitor cells located in proliferative zones produce a variety of neurons, thereby generating the diversity and complexity of neuronal phenotypes present in the adult brain (Jessell, 2000; Marquardt and Pfaff, 2001; Bertrand et al., 2002; Cheng et al., 2004; Götz and Huttner, 2005; Arlotta et al., 2008; Achim et al., 2014; Götz et al., 2016). Neural progenitors proliferate in the ventricular and subventricular zones of the developing forebrain, then exit the cell cycle and differentiate as they migrate away from these zones. During this process coordinated regulation of cell cycle exit and differentiation is essential for generation of the appropriate number of neurons and formation of the correct structural and functional connections of neuronal circuits. Previous studies have shown that the differentiation of neuronal progenitors is intimately connected with the regulation of the cell cycle and that the two processes are under coordinate control (Qian et al., 2000; Ohnuma and Harris, 2003; Hardwick et al., 2015).

Cend 1 is a neuronal-lineage specific modulator involved in timely coordination of cell cycle exit and differentiation of neuronal precursors (Koutmani et al., 2004; Georgopoulou et al., 2006; Politis et al., 2007a,b). Its expression is low, but clearly detectable, in precursor cells and is up-regulated upon neuronal differentiation, suggesting a functional role in the precursor to neuron switch. In agreement, gain- and loss-offunction experiments in neural stem/ progenitor cells showed that Cend1 prompts neuronal precursors to exit the cell cycle and differentiate to a neuronal phenotype (Katsimpardi et al., 2008; Makri et al., 2010). Further, genetic ablation of Cend1 in mice resulted in irregularities in cerebellar lamination caused by increased proliferation of granule cell precursors, delayed radial migration of granule cells and compromised Purkinje cell differentiation, particularly evident in stunted arborization of their dendritic tree (Sergaki et al., 2010). These alterations were associated with ataxic gait and deficits in motor coordination in Cend $1^{-/-}$mice.

To examine further the consequences of Cend1 genetic ablation, we determined herein a range of behaviors, including anxiety and exploratory behavior in the elevated plus maze (EPM), associative learning in fear conditioning, and spatial learning and memory in the Morris water maze (MWM). We observed significant deficits in all tests, suggesting structural and/or functional alterations in brain regions such as the cortex, amygdala and the hippocampus. In concurrence, immunohistochemistry revealed reduced numbers of $\gamma$-aminobutyric acid (GABA) GABAergic interneurons in the adult cerebral cortex and the amygdala, most notably in the basolateral nucleus, as well as aberrant neurogenesis in the dentate gyrus (DG) of the hippocampus.

GABAergic interneurons comprising about one fifth of the total neuronal population in the adult cortex, play a pivotal role in cortical circuitry and activity. They provide the principal source of inhibition to cortical circuits and regulate the activity of excitatory projection neurons (Lehmann et al., 2012; Kelsom and Lu, 2013). Previous studies have indicated that changes in the number, distribution and function of cortical interneurons are associated with a variety of severe neurological disorders, including autism, schizophrenia, epilepsy and anxiety (Matsuda et al., 2011; Le Magueresse and Monyer, 2013; Catavero et al., 2018). Cortical interneurons originate from the ganglionic eminence (GE), mostly the medial part medial GE (MGE), which is a distinct domain of the subpallial ventricular area, and migrate tangentially to reach the developing cortex where they reside (Marín and Rubenstein, 2003; Nakajima, 2007). The cellular and molecular mechanisms involved in interneuron generation and migration from their site of origin to the cortex have been studied extensively. The list of molecules involved includes both cell intrinsic cues-permissive or instructive-and cell extrinsic factors- attractive or repulsive (Crair, 1999; Vanderhaeghen and Polleux, 2004). Interestingly, cell cycle progression/exit of GE precursors has been linked to their normal migratory behavior (Vidaki et al., 2012) rendering Cend1 a potential player in this process.

Apart from cortical interneurons, the GE gives rise to GABAergic interneuron subtypes that contribute to the mammalian amygdala (Waclaw et al., 2010). This region is subdivided to the lateral, basolateral, and central amygdala and has been identified as a fundamental anatomic area of the circuitry processing fear conditioning (LeDoux, 2000, 2003). An important function of this region is to control behaviors that are related to fear and anxiety, in concert with the hippocampus and the cerebral cortex (Roozendaal et al., 2009), whose GABAergic interneurons also originate at the GEs (Nery et al., 2002). Notably, it has been reported that within this circuit inhibitory interneurons play a vital role in fear-related behaviors (Ehrlich et al., 2009).

In the present study we identified functional and cellular aberrations in Cend $1^{-/-}$mice, likely associated with reduced numbers of GABAergic, but not of glutamatergic, cells. We established that the paucity of GABAergic interneurons in the adult Cend $1^{-/-}$cortex and amygdala correlated with increased proliferation and apoptosis as well as with reduced migration of neuronal progenitors from the embryonic GE from which these cells originate. We further noted reduced hippocampal GABAergic neurons and aberrant neurogenesis in the adult DG of the hippocampus, both implicated in learning and memory processes. In the DG new neurons are produced continuously in the adult brain and become functionally integrated into pre-existing neuronal circuits thus contributing to behaviorally relevant neuronal assemblies (Eriksson et al., 1998; Kempermann et al., 2004; Parent, 2007; Trouche et al., 2009; Song et al., 2012; Nicola et al., 2015). An increasing number of studies indicates that adult hippocampal neurogenesis is functionally associated with hippocampal-dependent learning (Shors et al., 2001; Leuner et al., 2004; Snyder et al., 2005; Winocur et al., 2006; Kee et al., 2007; Kempermann et al., 2015). Moreover, adultgenerated hippocampal neurons are involved in spatial memory processes such as acquisition or retrieval, especially while still in an immature stage when they possess increased plasticity (Ramirez-Amaya et al., 2006; Tashiro et al., 2007; Abrous and Wojtowicz, 2015). Our analysis showed an activation of the earlier stages of adult hippocampal neurogenesis in Cend1-/mice, accompanied by increased cell apoptosis. These events may 
be causally related to a parallel decrease in local parvalbumin $(\mathrm{PV})$ interneurons, which are known to suppress neural stem cell activation (Song et al., 2012), but also to support the survival of newborn neurons (Song et al., 2013).Taken together, our data highlight the requirement for Cend 1 expression in the formation of a structurally and functionally normal phenotype.

\section{MATERIALS AND METHODS}

\section{Animals}

All international guidelines for animal care and use were applied in strict compliance with the European and National Laws for Laboratory Animal Use (Directive 2010/63/EU and Greek Law 56/2013), according to FELASA recommendations for euthanasia and the Guide for Care and Use of Laboratory Animals of the National Institutes of Health. All protocols were approved by the Animal Care and Use Committee of the Hellenic Pasteur Institute (Animal House Establishment Code: EL 25 BIO 013, License No. 4550/ 11-07-2014). The Cend $1^{-/-}$colony was maintained in the heterozygous state by backcrossing to C57BL/6J mice (Sergaki et al., 2010). Homozygous Cend1-/- adult animals or E14.5E16.5 embryos were produced by intercrossing heterozygous Cend1+/- mice. Genotyping was performed by PCR using two different sets of oligonucleotide primers: Forward $5^{\prime}$-CTAGAGAATTCAGGGAATTGGGGATG- $3^{\prime}$ and reverse $5^{\prime}$-AGTGTTGGACTCGTCCTCCTCTG- $3^{\prime}$ primers were used to amplify a 500-bp band within the Cend1 coding region present in wild-type (wt) and heterozygous animals and absent in knock-out mice. Forward 5'-CCGACGGCACGCTGATTGA$3^{\prime}$ and reverse $5^{\prime}$-GCTCCGCCGCCTTCATACTG-3' primers derived from the lacZ gene in the $\mathrm{pZ}$-lacz vector were used to amplify a 500-bp band present in heterozygous and knock-out mice and absent in wt animals.

\section{Behavioral Analyses}

Three different cohorts of animals were used for the behavioral analyses of Cend $1^{-/-}$adult male mice: one for the EPM $\left(10^{+/+}\right.$ and $15^{-/-}$animals), one for the cued fear conditioning $\left(8^{+/+}\right.$ and $8^{-/-}$animals $)$and one for the MWM test $\left(11^{+/+}\right.$and $14^{-/-}$ animals).

\section{Analysis of Anxiety Related Behavior Elevated Plus Maze (EPM)}

Mice were assessed between 09:00-12:00 h, during the light period of the daily cycle, in a room with moderate illumination of 150 lux. The apparatus encompassing two open and two closed arms, was elevated $60 \mathrm{~cm}$ above the ground. It was made of plastic, with a black-colored floor and gray-colored walls, $40 \mathrm{~cm}$ in height for the closed compartments. The central platform was $10 \times 10 \mathrm{~cm}$ in size and the arms $50 \times 10 \mathrm{~cm}$ in size. Each animal was placed in the center of the apparatus and it faced an open arm. Each animal was left to explore the EPM for $5 \mathrm{~min}$ and its movements were digitally recorded. For an animal to be considered within (entry) or outside (exit) an arm, all its limbs should be in the same arm. The following parameters were quantified independently by two investigators "blind" as to the group that animals belonged to: number of entries and time spent in both open arms, number of entries and time spent in both closed arms.

\section{Behavioral Analysis of Learning and Memory Function \\ Cued Fear Conditioning}

Before training, each animal was habituated for $3 \mathrm{~min}$ to the conditioning apparatus (black-colored box, Pansystems, $25 \times 25 \times 27 \mathrm{~cm}$ ). After the habituation period, the animal was exposed to a tone $(8 \mathrm{kHz}, 76 \mathrm{~dB})$ for 30 sco-terminating with an electric foot shock (intensity: $0.5 \mathrm{~mA}$; duration: $1 \mathrm{~s}$ ). This pairing was repeated three times. Assessment of cued fear memory was performed 8 days after training, in a modified conditioning apparatus: the floor grid metal bars delivering the shock were covered with a white cardboard and cut-out circles and rectangles were mounted on the walls of the conditioning box. In addition, novel olfactory cues (rose odor) had been introduced to the testing box. Each mouse received three 30-s tone presentations without shock, separated by $60 \mathrm{~s}$ intervals. Their behavior was analyzed off-line independently by two investigators "blind" as to the group the animals belonged to. Freezing was defined as absence of any other movement except the respiratory ones. The average time of freezing during the $30 \mathrm{~s}$ tones, was calculated for each animal.

\section{Morris Water Maze Test (MWM)}

The allocentric version of the MWM was employed, using a circular pool of $140 \mathrm{~cm}$ diameter. The pool was filled with water $\left(24 \pm 1^{\circ} \mathrm{C}\right)$; in order to make the water opaque, a non-toxic, water-soluble white dye was added to the water. Mice were trained for 5 consecutive days and $24 \mathrm{~h}$ later they were exposed to a memory probe trial. During training, mice had to locate a hidden platform $(8 \mathrm{~cm} \times 10 \mathrm{~cm}$ made of transparent plastic $)$ positioned $1 \mathrm{~cm}$ under the water surface; the position of the platform was fixed relative to extra-maze visual cues. Each day animals were exposed to four trials (each trial had a maximum duration of $60 \mathrm{~s}$ ), separated by 15 min intervals. In each trial the starting position was different, in a pseudorandom order. Each trial ended when the animal found the platform or after $60 \mathrm{~s}$ in which case the animal was placed by the experimenter on the platform. In either case, it remained on the platform for $20 \mathrm{~s}$ and then it was returned in its home cage. Memory probe trial took place $24 \mathrm{~h}$ after the last training trial. For the probe trial, the platform was removed, the animal entered the water maze from a point opposite to the quadrant of the maze were the platform used to be during training and it was allowed to explore the maze for $60 \mathrm{~s}$. The Noldus Ethovision system (Ethovision 3.0, Noldus Information Technologies, Wageningen, Netherlands) was use for the documentation and analyses of the behavior of mice during the training and memory probe trials. For training, for each mouse latency (time in sec to mount the platform) was determined for each trial and an average was calculated for each day. For the probe trial, we calculated the time spent in the target quadrant as well as that spent in the opposite quadrant. In order to exclude the possibility that any learning or memory deficits observed in the MWM were 
due to deficits in motor activity, swim speed $(\mathrm{cm} / \mathrm{sec})$ was also calculated.

\section{Tissue Preparation and Immunofluorescence Procedures}

Mice (3-4 per genotype) were euthanized by isoflurane inhalation and perfused transcardially with $4 \%$ paraformaldehyde fixative in phosphate-buffered saline (PBS). Following dissection, brains were post-fixed at $4^{\circ} \mathrm{C}$ overnight and cryoprotected for 2 days at $4^{\circ} \mathrm{C}$ in sucrose (30\% w/v in PBS). Tissues were then embedded in O.C.T. compound (VWR Chemicals) and frozen or in some cases processed for vibratome sectioning $(50 \mu \mathrm{m})$, as indicated. Series of coronal cryosections $20 \mu \mathrm{m}$-thick were cut and kept at $-20^{\circ} \mathrm{C}$ until processed for immunohistochemistry. After thawing sections were treated for $1 \mathrm{~h}$ with $5 \%$ normal donkey serum (NDS) to block non-specific binding sites and permeabilized with $0.2 \% \mathrm{v} / \mathrm{v}$ Triton $\mathrm{X}-100$ in PBS. Overnight incubation with primary antibodies diluted in $2.5 \%$ NDS in $\mathrm{PBS}$ at $4^{\circ} \mathrm{C}$ was followed by incubation with secondary antibodies for $2 \mathrm{~h}$ at room temperature. Primary antibodies used were: rabbit polyclonal anti-GABA (1:100; Sigma) for GABAergic neurons; rat monoclonal anti-somatostatin (anti-Sst; 1:1,000; Millipore) for GABAergic interneuron subtype; mouse monoclonal anti-Satb2 (1:300; Abcam) for glutamatergic interneurons; rabbit polyclonal anti-calbindin (1:1,000; Chemicon) and rabbit polyclonal anti-PV (1:1,000; Swant) for calcium binding proteins; anti-phosphohistone 3 (PH3; 1:500, Upstate) and mouse monoclonal anti-Ki67 (1:400; Beckton Dickinson) for mitotic and proliferating cells, respectively; rabbit polyclonal anti-caspase 3 (1:400; Cell Signaling) for apoptosis; mouse monoclonal anti-glial fibrillary acidic protein (GFAP, 1:500; Sigma) for parenchymal astrocytes and neural stem cells in the subgranular layer (SGL) of the DG; rabbit polyclonal anti-Sox2 (1:200; Abcam) for neural stem and progenitor cells in the subgranular zone (SGZ) of the DG; goat polyclonal anti-doublecortin (DCX; 1:50; Santa Cruz); mouse monoclonal anti-NeuN (1:300; Chemicon) and rabbit polyclonal anti-Prox1 (1:200; Abcam) for newborn and mature DG neurons; rabbit polyclonal anti-Ibal (1:200; Wako) and rat monoclonal anti-CD68 (1:150; Serotec) for total and activated microglia/macrophages; mouse monoclonal anti-Mash1 (1:50; BD Pharmigen) for identification of GABAergic interneuron precursors in the GEs. Secondary antibodies conjugated to Alexa Fluor 488 or 546 and TO-PRO-3 (1:1,000) used to visualize cell nuclei were from Molecular Probes. Sections were mounted on Prolong Gold antifade (Molecular Probes) and confocal microscopy was performed in Leica TCS SP and Leica TCS-SP5II confocal microscopes.

\section{Image Analysis}

\section{Cell Counts on Embryo and Adult Brain}

For each animal, digital images from 3-4 brain sections were acquired in a confocal microscope and the number of cells positive for a specific marker were counted using ImageJ areas of interest, "blindly" as to the group animals belonged to, and setting the threshold at a constant value. The brain areas analyzed include the adult somatosensory cortex, the basolateral amygdala (BLA) and the hippocampal DG as well as the embryonic GEs. Counts were averaged from three fields per area and per animal ( $n=3$ mice per genotype).

\section{Fluorescence Intensity}

For evaluation of GFAP expression in the parenchyma of the DG, fluorescence intensity (pixels) was measured as previously described (Papastefanaki et al., 2015; Terzenidou et al., 2017). Briefly, single channel stacks of confocal images were acquired under the same settings (constant gain and offset values, $3 \times$ averaging, $1024 \times 1024$ resolution, $1-\mu \mathrm{m}$ step size). Quantification of fluorescence intensity was performed using Image J software by a blind observer, after free-hand selection of the region of interest (ROI) and setting the threshold at a constant value. Measurements on each single image of the confocal stack were added up and normalized to the area of the ROI. Three animals were analyzed per genotype and the values from three sections were averaged per mouse.

\section{Automated Calculation of the Density of Satb2+ Cells} In the first step of analysis, the Imaris "spots" module was used to calculate automatically the total number of nuclei (estimated diameter $>6 \mu \mathrm{m}$ ) in the area of the somatosensory cortex. In the second step, only the nuclei that were positive for Satb2 were selected, using the "filter" module of spots (filter type: max intensity of green channel for Satb2). Finally, the number of Satb2 ${ }^{+}$cells was calculated as a percentage of total nuclei, and setting the threshold at a constant value. Counts were averaged from three fields per area and per animal $(n=3$ mice per genotype).

\section{Preparation of Organotypic Slice Cultures}

Slice cultures were prepared as previously described (Lavdas et al., 1999; Kouroupi et al., 2010). Briefly, pregnant C57BL6 mice at two different stages of gestation (E14, $n=3$; E16, $n=3$ ) were euthanized by isoflurane inhalation. The fetuses were immediately removed and immersed in sterile HBSS at $4^{\circ} \mathrm{C}$ containing $6.5 \mathrm{mg} / \mathrm{ml}$ glucose. All following procedures were performed under sterile conditions. Brains were removed and embedded in 3\% agarose/ $0.1 \mathrm{M}$ PBS, $\mathrm{pH}$ 7.2. Coronal slices (400 $\mu \mathrm{m}$-thick) were cut using a Leica vibrating microtome and kept for $45 \mathrm{~min}$ at $4^{\circ} \mathrm{C}$ in HBSS/glucose to allow for decline of enzymatic activity released by damaged cells. Slices were placed onto millicell $\mathrm{CM}$ membranes in $30 \mathrm{~mm}$ Petri dishes containing $1 \mathrm{ml}$ of DMEM/F12 supplemented with $6.5 \mathrm{mg} / \mathrm{ml}$ glucose $/ 0.1 \mathrm{mM}$ glutamine $/ 50 \mathrm{mg} / \mathrm{ml}$ penicillin/streptomycin/10\% FCS. After $1 \mathrm{~h}$ the medium was changed to Neurobasal supplemented with B27 (1:50) and N2 (1:100) containing $6.5 \mathrm{mg} / \mathrm{ml}$ glucose $/ 0.1 \mathrm{mM}$ glutamine $/ 50 \mathrm{mg} / \mathrm{ml}$ penicillin/streptomycin.

\section{Administration of Fluorescent Tracer}

To investigate neuronal migration, we used a glass micropipette to place DiI crystals (Molecular Probes; Lavdas et al., 1999; E14, $n=3$; E16, $n=5$ ) on one hemisphere of cultured slices. After 
tracer placement, slices were cultured in Neurobasal medium for a further $48 \mathrm{~h}$ and were then fixed for $3 \mathrm{~h}$ in $4 \%$ formaldehyde in PBS. Slices were subsequently rinsed in PBS, mounted on microscope slides, and observed with a laser-scanning confocal microscope (Leica TCS SP).

\section{Statistical Analyses}

The effect of genotype on the latency to reach the platform during the learning period in the MWM was assessed using one-way ANOVA with repeated measures (day) and genotype as the independent factor. The effect of genotype on time spent in the target and opposite quadrants of the MWM during the long-term memory probe trial was assessed using a two-way ANOVA with genotype and quadrant as independent factors. In the case of significant interactions, univariate $F$ tests were used to evaluate the main effects. Finally, the effect of genotype on all other behavioral parameters was assessed using one-way ANOVA, with genotype as the independent factor. For statistical comparisons of the immunohistochemical data between genotypes the two-tailed unpaired Student's $t$-test was used. All statistical analyses were performed using the statistical software SPSS 21 for Windows. Values are expressed as mean \pm standard error of mean (SEM) and significance is defined as ${ }^{*} p<0.05$; ${ }^{* *} p<0.01$; $^{* * *} p<0.001$ for genotype differences and \#\#\# $p<0.001$ for the difference in the time spent in the target and opposite quadrants during the probe trial of MWM.

\section{RESULTS}

\section{Cend1-/- Mice Exhibit Severe Behavioral Deficits}

To investigate the effects of Cend1 genetic ablation, we exposed Cend $1^{-/-}$mice to a repertoire of behavioral tests. First, we assessed anxiety related behavior in the EPM, where Cend $1^{-/-}$ mice spent less time in and made fewer entries into the open arms compared to wt animals, indicative of higher levels of anxiety. More specifically, for both the time spent in the open arms and the number of entries into the open arms, statistical analysis revealed a significant genotype effect: $F_{(1,24)}=9.994, p=0.004$ and $F_{(1,24)}=12.621, p=0.002$, respectively (Figures 1A,B). Notably, no difference was observed in the number of entries into the closed arms $\left(F_{(1,24)}=0.517\right.$, $p=0.479)$.

Cend $1^{-/-}$mice also showed a mild but statistically significant deficit in cued fear memory 8 days following fear conditioning $\left(F_{(1,15)}=8.684, \quad p=0.011\right.$; Figure 1C). It should be noted that during fear conditioning training, both Cend $1^{-/-}$ and wt mice oriented towards the source of the tone (i.e., could detect the tone) and they showed the normally expected response to the noxious stimulus of the electric foot shock.

Finally, mice were assessed for spatial learning and memory in the MWM, a hippocampal-dependent task which is also used to evaluate possible damage to cortical regions of the brain (D'Hooge and De Deyn, 2001). Adult mice went through a training phase during which they were evaluated for their learning ability by measuring the latency to find a hidden platform. All mice, either wt or Cend1 ${ }^{-/-}$, had a normal swimming speed and no difficulty to climb on the submerged escape platform. It should be mentioned that similarly to our observations, in other models of ataxic mice, ataxia did not affect the swimming speed of the animals possibly due to the nature of this measurement: gait-impaired animals can apparently compensate in the water with other body movements or with extra hind-limb kicks (Lalonde and Botez, 1986; Savvaki et al., 2008; Jayabal et al., 2015).

Nevertheless, a significant day $\times$ genotype interaction was observed on the latency to find the hidden platform $\left(F_{(4,92)}=3.955, p=0.005\right)$. Further analysis indicated that wt animals showed the normal improvement in their performance across days (progressively reduced latency to find the hidden platform) while Cend $1^{-/}$mice failed to do so. More specifically, on days 4 and 5 of training, Cend $1^{-/-}$animals showed statistically significant higher latencies to reach the hidden platform compared to wt mice ( $p=0.045$ for Day 4 and $p=0.005$ for Day 5; Figure 1D). In the long-term memory probe trial ( $24 \mathrm{~h}$ after the last training trial), statistical analysis revealed a significant quadrant $\times$ genotype interaction on the time spent in the target and opposite quadrants $\left(F_{(1,49)}=8.382\right.$, $p=0.006$; Figure 1E). As expected, given their poor learning performance, Cend $1^{-/-}$mice showed no preference for the target quadrant (post hoc test $p=0.452$ ), while wt animals exhibited a clear preference spending more time in the target quadrant as compared to the opposite one (post hoc test $p<0.001)$.

\section{Cend1-/- Mice Have Reduced Number of Interneurons in the Adult Cortex and Amygdala}

We next sought to investigate whether the behavioral phenotype of Cend $1^{-/-}$mice is accompanied by cellular changes in specific brain regions that are functionally relevant. It has been shown that increased levels of anxiety are related to a reduction in the GABAergic population, resulting in decreased inhibitory neurotransmission in the cortex (Löw et al., 2000). In agreement, immunofluorescence labeling for the neurotransmitter GABA in coronal cortical sections from adult mice revealed a decrease in immunolabeled cells in Cend1 $1^{-/}$mice as compared to wt, particularly in the somatosensory cortex (Figure 2A). Quantification demonstrated a specific, statistically significant reduction in the density of GABAergic neurons (53\% reduction, $P=0.0007$ ) in this region (Figure 2B) whilst the density of glutamatergic neurons identified by Satb2 immunofluorescence, was not changed in Cend $1^{-/}$mice (Supplementary Figure S1). We further examined the presence of interneuron subtypes in the adult cortex by immunofluorescence for the calciumbinding proteins $\mathrm{PV}$ and calbindin. We noted a prominent reduction $(49 \%, P=0.003)$ in the number of $\mathrm{PV}$-expressing interneurons (Figures 2C,D) of Cend $1^{-/}$mice as compared to their wt littermates, as well as a significant reduction $(22 \%$ reduction, $P=0.008$ ) in the number of calbindin ${ }^{+}$interneurons 
Behavior in the Elevated Plus Maze

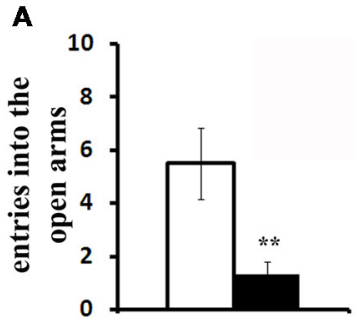

B

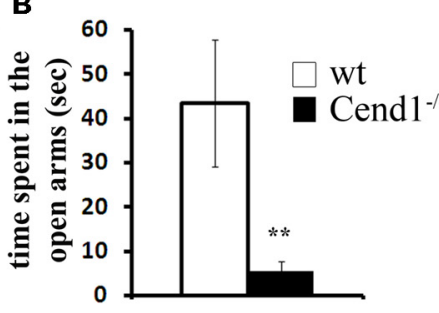

\section{Cued Fear Memory}

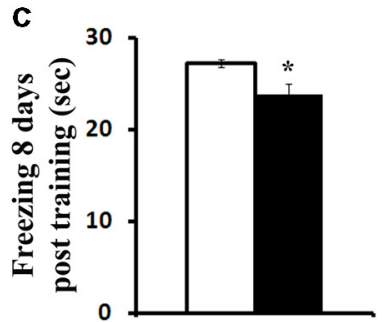

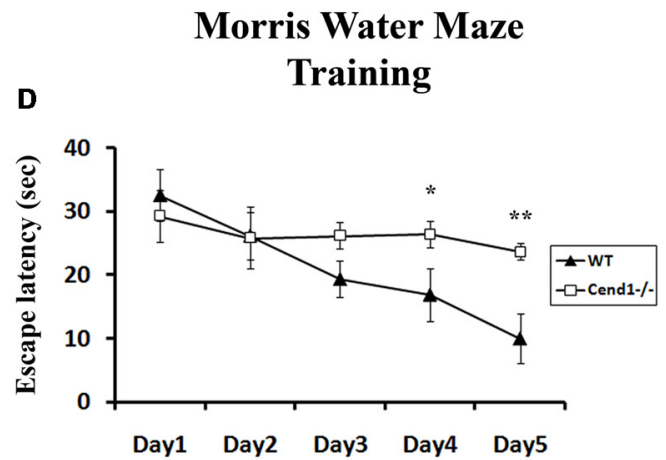

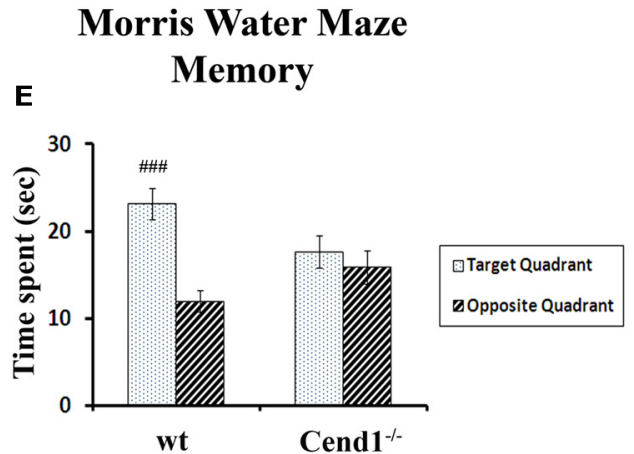

FIGURE 1 | Behavioral deficits in adult Cend1 ${ }^{-/-}$mice. Cend1 $1^{-/-}$mice show increased anxiety as assessed in the elevated plus maze (EPM) task, making fewer entries (A) and spending less time in the open arms of the maze (B) than their wild-type (wt) littermates ( $n=10$ for wt mice and $n=15$ for Cend $1^{-/-}$mice). Cend1 ${ }^{-/-}$mice show a mild deficit in cued fear memory 8 days following fear conditioning (C) as compared to wt animals $(n=8$ for both genotypes), as well as severe cognitive deficits in the Morris water maze (MWM) test, both during learning (D) and in the memory trials (E; $n=11$ for wt mice and $n=14$ for Cend $1^{-/-}$ mice). Values represent mean \pm standard error of mean (SEM); ${ }^{*} P<0.05$; ${ }^{* *} P<0.01$; \#\#\#, $P<0.001$.

(Figures 2E,F). Finally, Sst-expressing interneurons that have a much lower abundance than the other two interneuron subtypes, were also significantly reduced $(63 \%$ reduction, $P=0.031$; Figure 3).

Apart from the cortex, the behavioral phenotype of Cend $1^{-/-}$ mice pointed to possible cellular alterations in the amygdala, a region associated with fear and anxiety-related behavior along with the cerebral cortex. Previous studies have shown that a reduction in the GABAergic population residing in the amygdala (Waclaw et al., 2010) correlated with increased levels of anxiety (LeDoux, 2003), with the BLA and central nuclei being especially involved in the amygdalar circuitry that mediates control of anxiety (Tye et al., 2011). Based on these observations, we examined by immunofluorescence labeling the GABAergic population in the amygdala of $\mathrm{Cend}^{-/-}$and wt mice and found a severely compromised population of GABAergic neurons (62\% reduction, $P=0.008$ ) in the mutant mice (Figures $4 \mathrm{~A}, \mathbf{B}$ ). Examination of interneuron subtypes revealed a statistically significant decrease in the number of calbindin ${ }^{+}$cells in Cend $1^{-/-}$mice (41\% reduction, $P=0.024$ ) as compared to wt (Figures 4Ca-f,D), particularly in the basolateral nucleus (BLA). However, unlike the somatosensory cortex, we did not observe a similar reduction in the $\mathrm{PV}^{+}$population in this brain area (Figures 4Ca,d,D).

\section{Reduced Migration of MGE-derived GABAergic Progenitors Is Associated With Altered Cell Proliferation and Apoptosis in Cend1-/- Mouse Embryos}

To evaluate the origin of the cellular changes in the GABAergic population observed in the adult cortex and the amygdala of $\mathrm{Cend}^{-/-}$mice, we questioned what happens during development that could possibly lead to such a defect. To address this, we investigated the generation of GABAergic interneurons in the medial embryonic GE which gives rise to cortical GABAergic interneurons, but also to a population of interneurons of the adult amygdala. Fluorescent tracer experiments have previously shown that cells deriving from the MGE migrate to the developing cortex (Lavdas et al., 1999). To investigate whether Cend1 deletion affects the migratory behavior of MGE-derived interneurons, we used a similar approach by placing DiI crystals in the MGE of slice explants cultures prepared from the brains 


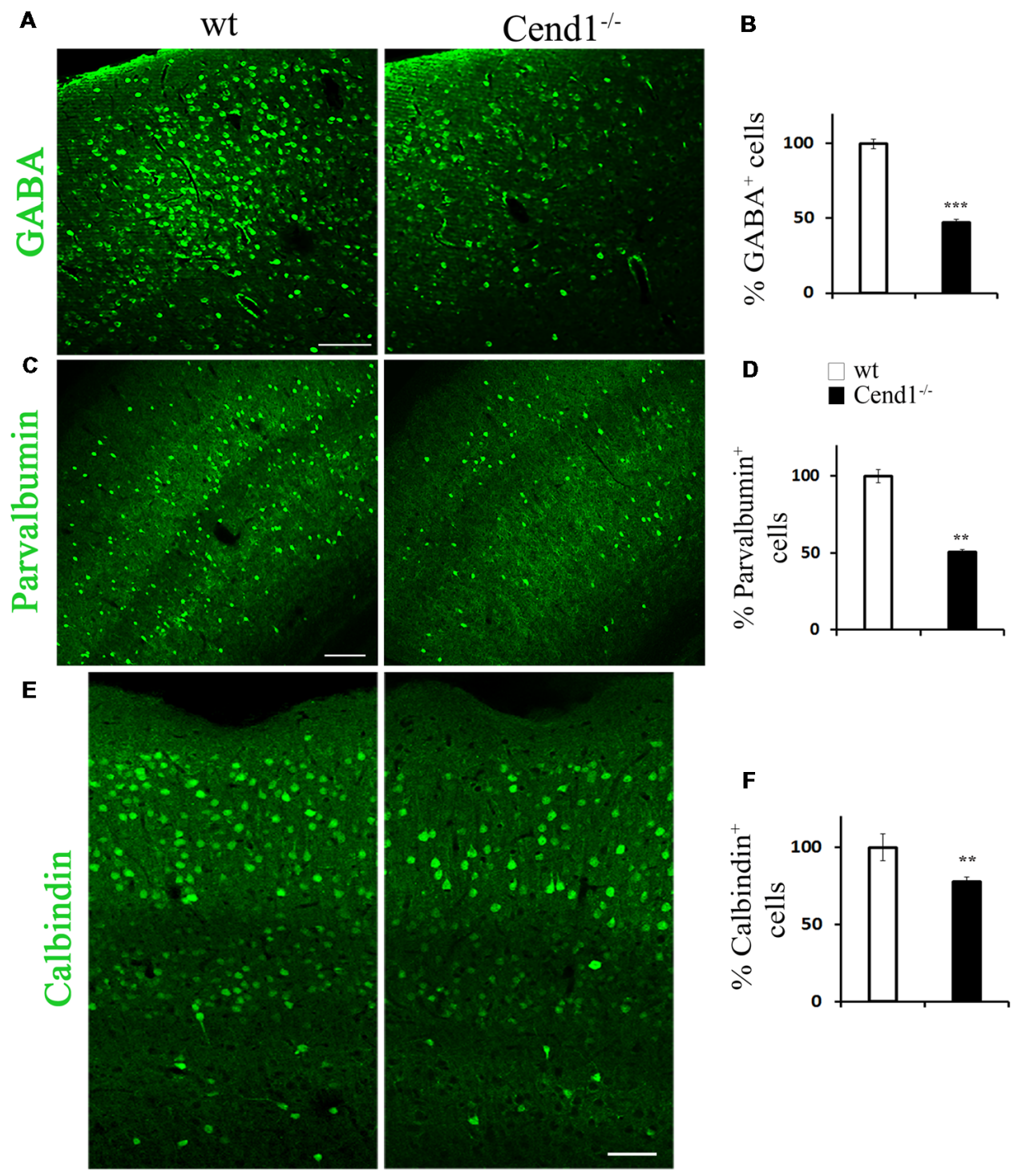

FIGURE 2 | Reduced interneurons in the adult cortex of Cend1-/- mice. Cryostat sections of adult somatosensory cortex immunostained for $\gamma$-aminobutyric acid (GABA; $\mathbf{A})$, parvalbumin (PV; C) and calbindin (E). Percentage of GABA ${ }^{+}$interneurons is reduced by half in Cend1 $1^{-/-}$mice by comparison to wt animals (B). This is mainly reflected in reduction of $\mathrm{PV}^{+}$cells (D) and to a lesser extent in reduction of calbindin ${ }^{+}$cells $(\mathbf{F})$ in Cend1 ${ }^{-1-}$ mice as compared to wt animals $(n=3$ per genotype). Values represent mean \pm SEM. Scale bars (A,C), $100 \mu \mathrm{m} ;(\mathbf{E}), 60 \mu \mathrm{m} ;{ }^{* *} P<0.01 ;{ }^{* * *} P<0.005$.

of mouse embryos at embryonic days E14.5 (Figure 5A) and E16.5 (Figure 5B). After further incubation for $48 \mathrm{~h}$, $\mathrm{DiI}^{+}$cells that had migrated outside the core of labeled cells were seen in both wt and Cend $1^{-/-}$mice. However, the numbers of migrating cortical progenitors were reduced almost by half in Cend $1^{-/-}$mice, at both developmental stages (for E14.5 $P=0.003$ and for E16.5 $P=0.02$; Figure 5C).

We then asked if the reduction in migrating progenitors is due to perturbed progenitor cell proliferation in the MGE. Immunofluorescence labeling of E14.5 coronal forebrain sections with the mitotic marker $\mathrm{PH} 3$ and quantification revealed a statistically significant increase $(40 \%$ increase, $P=0.041)$ in the number of cells undergoing mitosis in the GE of Cend1 $1^{-/-}$ mice when compared to their wt littermates (Figures 5D,E). Thus, in the absence of Cend1, fewer cells are likely to exit the cell cycle and start their trajectory to the cortex. We then examined the expression of Mash1 (Ascl1), a transcription factor with a pivotal role in the generation and specification of GABAergic interneurons (Castro et al., 2011; Xu et al., 2014). Double immunofluorescence labeling for Mash1 and the proliferation marker Ki67, revealed a significant increase in both the total number of proliferating $\mathrm{Ki}^{+} 7^{+}$cells $(21 \%$ increase, $P=0.015$; Figures $\mathbf{5 F}, \mathbf{G})$ as well as in the cycling Mash $1^{+} / \mathrm{Ki}^{+} 7^{+}$neuronal progenitors $(38 \%$ reduction, $P=0.014$; Figures $5 \mathbf{F}, \mathbf{H})$ in the MGE of Cend $1^{-/-}$mice. Concomitantly, 

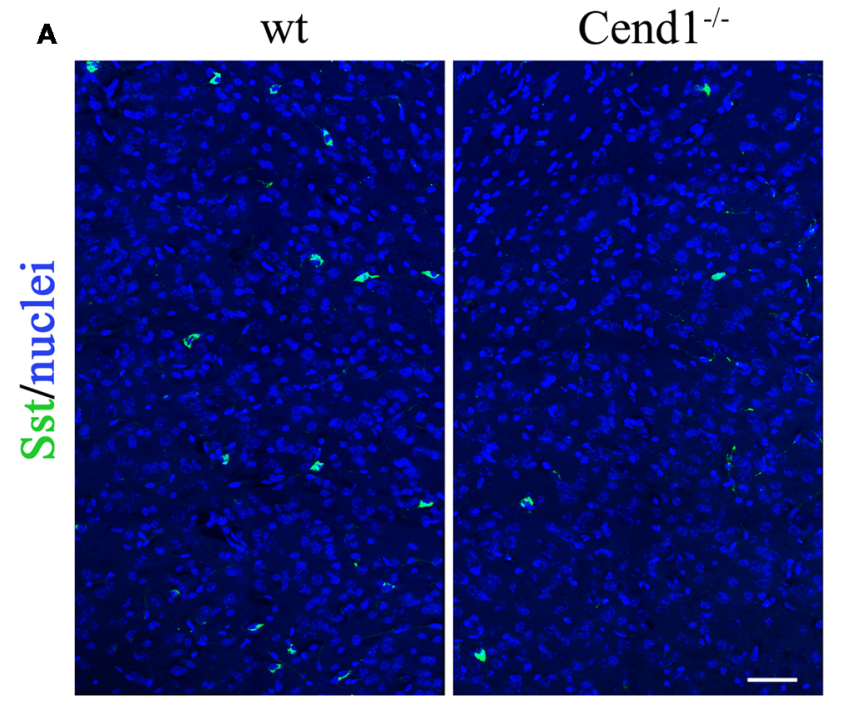

B

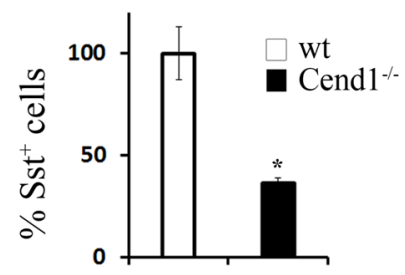

FIGURE 3 | Decreased somatostatin (Sst)-expressing interneurons in the adult cortex of Cend1-1- mice. Confocal images of cryostat sections of adult somatosensory cortex immunostained for Sst (A) and quantification of Sst ${ }^{+}$cells in Cend $1^{-/-}$mice as compared to wt animals $(\mathbf{B} ; n=3$ per genotype). Values represent mean \pm SEM. Scale bar (A), $50 \mu \mathrm{m} ;{ }^{*} P<0.05$.

immunofluorescence labeling for activated caspase-3 as an apoptotic marker, revealed a statistically significant increase (41\% increase, $P=0.035$ ) in apoptotic cell death in the GE of Cend $1^{-/}$mice as compared to wt littermates (Figures 5I,J). The combined phenomena of increased cellular proliferation in germinal zones accompanied by increased cell death and reduced migratory activity could contribute to the lower number of GABAergic interneurons in the cortex and amygdala of adult Cend $1^{-/-}$mice.

\section{Reduced Interneurons and Aberrant Neurogenesis in the Hippocampus of Adult Cend1-/- Mice}

As adult hippocampal neurogenesis is intimately related to higher cognitive functions, most notably learning and memory processes (Kempermann et al., 2015), we examined if the cognitive deficit noted in Cend $1^{-/-}$mice is associated with cellular alterations in the SGZ of the adult DG, which constitutes the adult hippocampal neurogenic niche. $\mathrm{GFAP}^{+}$and/or Sox $2^{+}$ neural stem and progenitor cells residing in the SGZ of the DG generate proliferating $\mathrm{DCX}^{+}$neuroblasts that give rise to post-mitotic $\mathrm{DCX}^{+}$newborn neurons, which subsequently differentiate into granule neurons. Immunofluorescence for the general neuronal marker $\mathrm{NeuN}$ and the transcription factor Prox1 that specifically marks DG granule neurons, did not show any overt differences between genotypes (Figures 6A-D). However, there was a significant decrease in PV-expressing interneurons in the DG of Cend $1^{-/-}$mice (53\% reduction, $P=0.0059$; Figures 6E-G). Interestingly these local interneurons (basket cells), which are located in the immediate vicinity of the SGZ, have a dual function: on one hand they are known to suppress the activation of quiescent neural stem cells through GABA release, and on the other to control the survival of newborn neurons in the hippocampal neurogenic niche (Song et al., 2012, 2013; Saleem et al., 2013).

To examine if the reduction in PV interneurons was paralleled by an increase in neural stem and precursor cells in the SGZ, we performed double labeling for GFAP and Sox2. Surprisingly, we noted an increase in GFAP immunoreactivity (37\% increase, $P=0.0001$; Figures $7 \mathbf{A}-\mathbf{C}$ ) as well as in the number of Sox $2^{+}$cells $(24 \%, P=0.0002)$ throughout the DG, particularly within the hilus, suggesting astroglial activation (Figures 7D-F). To check if this was a sign of astrogliosis accompanied by microgliosis and inflammation, we immunolabeled for the ionized calcium-binding adapter molecule 1 (Iba1) and CD68, to detect total and activated microglial cells, respectively. However, we could not find evidence for inflammatory astro- and/or microgliosis, as we did not detect any differences between genotypes (Supplementary Figure S2).

Local astrocytes play a key role in promoting neurogenesis (Kempermann et al., 2015). To address if the increased levels of astrocytes in concert with the reduction in local PV interneurons affected neurogenesis, we next quantified the numbers of $\mathrm{GFAP}^{+} / \mathrm{Sox}^{+}$neural stem cells and $\mathrm{GFAP}^{-} /$Sox $^{+}$ progenitor cells lying in the SGZ (Figures 7G-I). We found a significant increase of both cell types in Cend $1^{-/-}$mice $(40 \%$, $P=0.0004$ and $35 \%, P=0.0002$, respectively), suggesting an expansion of the neural stem/progenitor cell pool. This was further supported by an increase in SGZ cell proliferation (39\%, $\left.P=3 \times 10^{-6}\right)$ in Cend1 $1^{-/-}$mice as indicated by Ki67 labeling (Figures 7J-L), as well as by an increase in $\mathrm{DCX}^{+} / \mathrm{Ki}^{+} 7^{+}$cycling neuroblasts $\left(36 \%, P=5.3 \times 10^{-5}\right)$ and in $\mathrm{DCX}^{+} / \mathrm{Ki}^{-} 7^{-}$newborn neurons $\left(31 \%, P=3.39 \times 10^{-5}\right.$; Figures $\left.7 \mathbf{M}-\mathbf{O}\right)$. Concomitantly, there was a significant increase in cell apoptosis $(60 \%, P=0.006$; Figures 7P-R). 

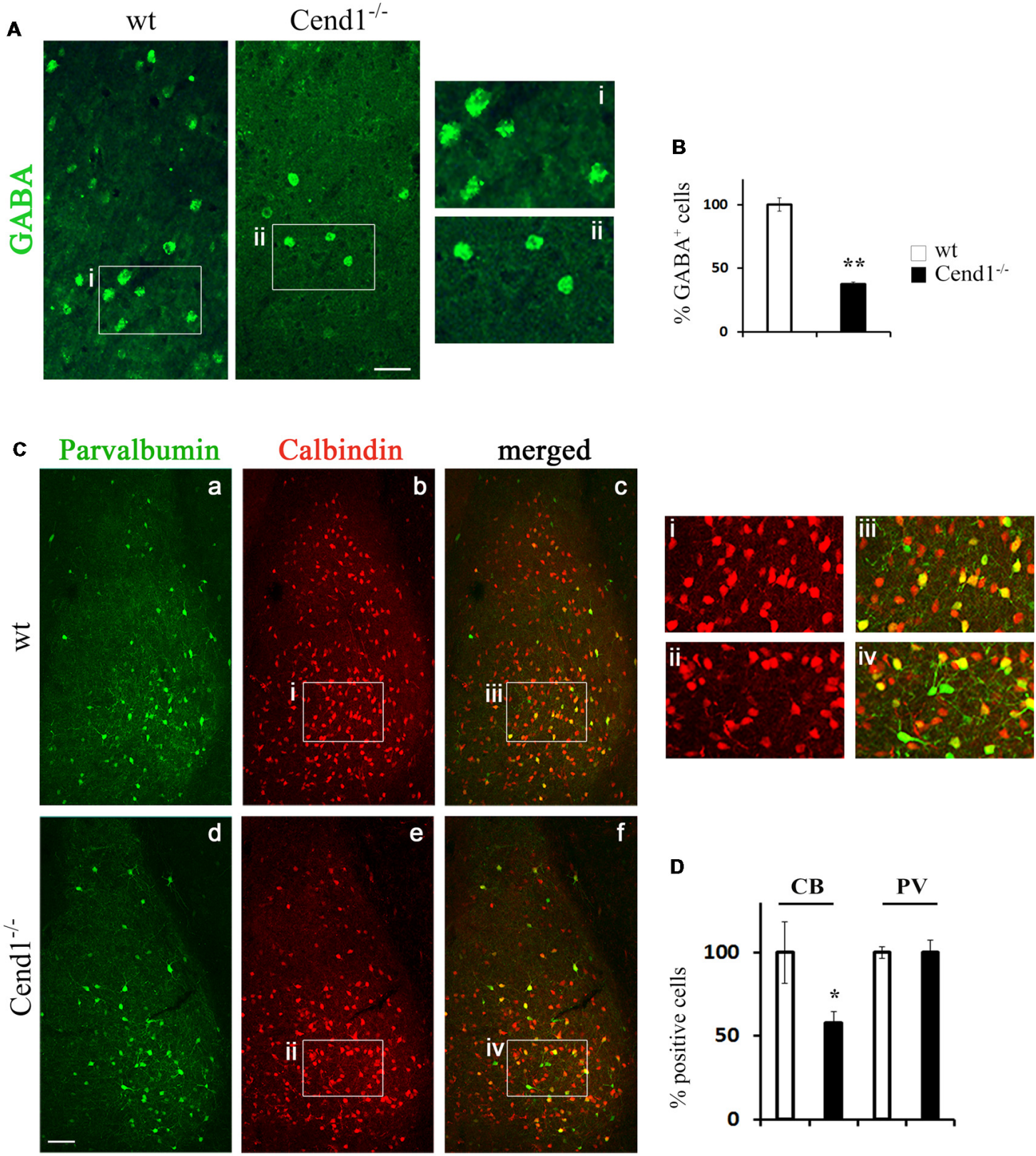

FIGURE 4 | Reduced interneurons in the adult amygdala of Cend1-/- mice. Confocal images of vibratome sections of adult amygdala immunostained for GABA (A) and quantification of GABA ${ }^{+}$cells in Cend1 $1^{-/-}$mice as compared to wt animals (B). The insets in (A) are shown at higher magnification in (Ai-Aii). (C) Double immunofluorescence labeling for PV (green; $\mathbf{C a}, \mathbf{d}$ ) and calbindin (red; $\mathbf{C b}, \mathbf{e}$ ) followed by confocal imaging shows partial overlap of the two populations (Cc,f). The insets in (C) are shown at higher magnification in (Ci-Civ). (D) Quantification shows that PV ${ }^{+}$cells do not differ between wt and Cend1-/- mice while calbindin ${ }^{+}$cells are reduced almost by half in Cend $1^{-/-}$mice $\left(n=3\right.$ mice per genotype). Values represent mean \pm SEM. Scale bar $40 \mu \mathrm{m}$ (A), $100 \mu \mathrm{m}$ (Cd); $P<0.05 ;{ }^{* *} P<0.01$.

Overall our analysis on adult hippocampal neurogenesis demonstrated that the earlier stages of this complex process, which include neural stem and progenitor cell activation as well as neuroblast and newborn neuron generation, are enhanced in the absence of Cend1. However at later stages, an increased number of cells are eliminated by apoptotic death, probably due to insufficient support provided by the reduced numbers of PV interneurons.
Finally, the reduction in PV interneurons noted in the DG prompted us to ask if there are also alterations in the abundance of these cells, as well as of Sst-expressing interneurons, all over the hippocampus. This is an important question given the involvement of these interneuron subtypes in memory processes (Murray et al., 2011; Ognjanovski et al., 2017). Accordingly, we found a reduction in both interneuron subpopulations in the CA1 -3 regions of Cend $1^{-/-}$mice (41\% reduction, $P=0.0054$ for 

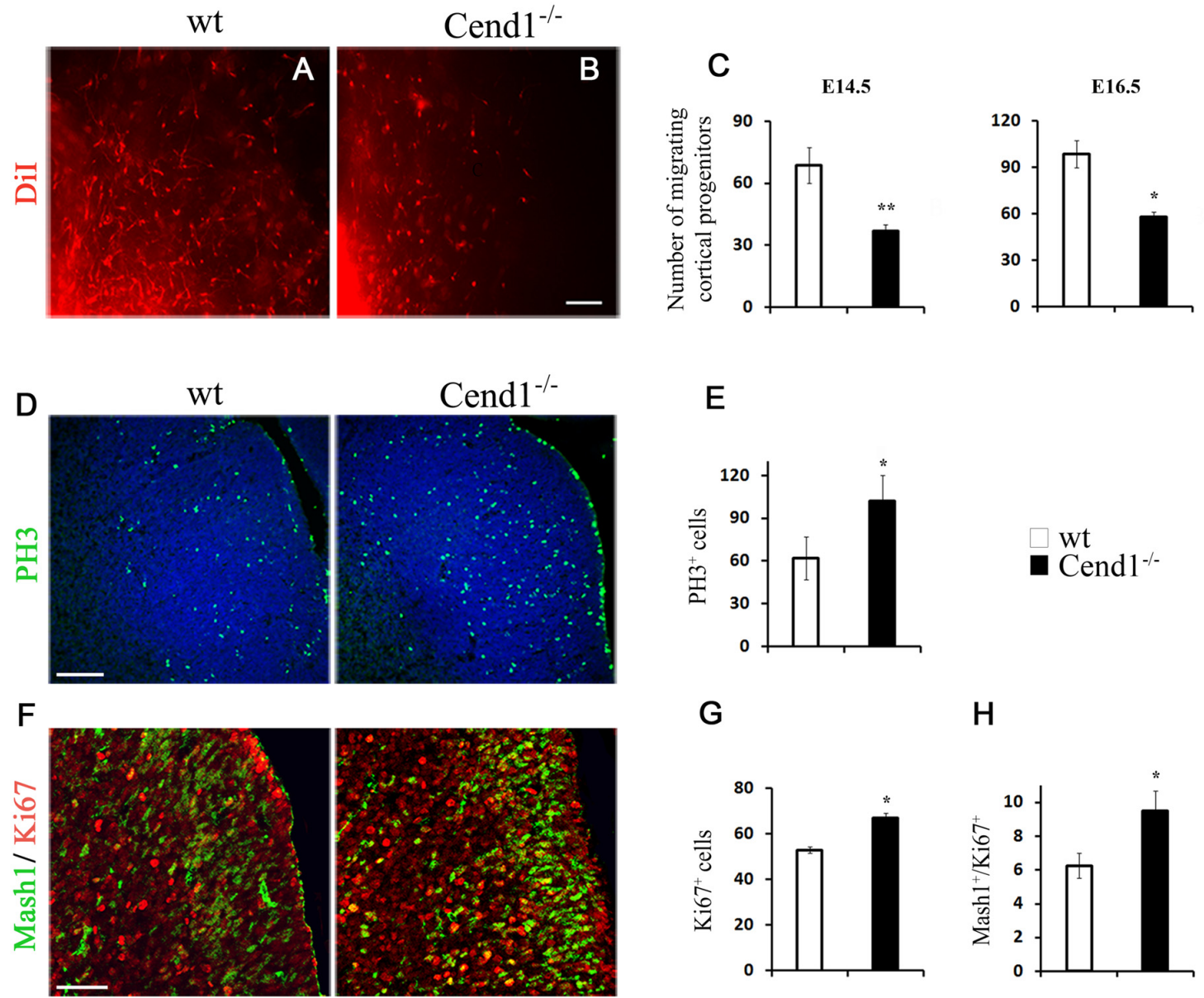

G

$\mathrm{H}$
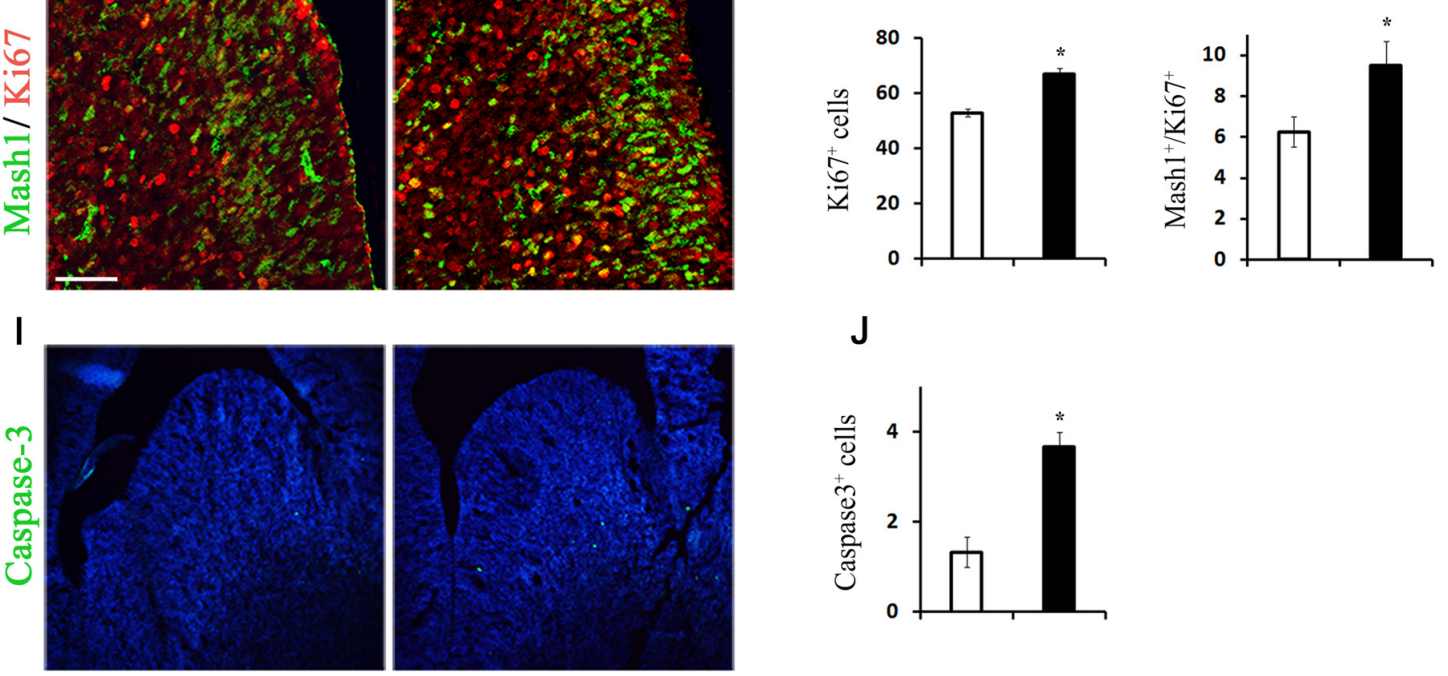

FIGURE 5 | Reduced migration and altered cell proliferation and apoptosis in Cend1 ${ }^{-/-}$mouse embryos. (A,B) Live images of Dil-labeled wt (A) and Cend1 ${ }^{-/-}$(B) organotypic slices from E16.5 mouse forebrain after 2 days in vitro. At the bottom left, shown is the edge of the Dil crystal placed on the medial ganglionic eminence (MGE) to label migrating GABAergic interneuron precursors. (C) Quantification of Dil-labeled cells at the beginning of the "stream" adjacent to the MGE in E14.5 and E16.5 slices reveals significantly decreased numbers of migrating cortical progenitors in Cend1-1- mice [E14.5: $68.7 \pm 8.3$ cells in wt $(n=4)$ vs. $37 \pm 2$ in Cend1- ${ }^{-1-}$ mice $(n=3)$; E16.5: $98.4 \pm 9.7$ cells in wt $(n=5)$ vs. $58.0 \pm 10.1$ in Cend1 $1^{-/-}$mice $(n=4)$ ]. (D) Immunofluorescence for the mitotic marker phosphohistone 3 (PH3) in cryostat sections of E14.5 MGE and quantification of labeled cells in (E), show significantly increased proliferation in Cend1 $1^{-/-}$mice $(62.0 \pm 14.8$ cells in wtvs.102.3 \pm 24.7 in Cend1 ${ }^{-1-}$ mice, $n=4$ per genotype). (F) Double immunofluorescence labeling for Mash1 (green) and Ki67 (red) in cryostat sections of E14.5 MGE. Quantification of immunolabeled cells reveals a significant increase in the number of proliferating Ki67 ${ }^{+}$cells (G; $53.0 \pm 1.5$ in wt: vs. $\left.67.0 \pm 2.\right)$, as well as in cycling Mash $1^{+} / \mathrm{Ki}_{6} 7^{+}$neuronal progenitors $\left(\mathbf{H} ; 6.0 \pm 0.7 \mathrm{in}\right.$ wt vs. $9.75 \pm 0.85$ in Cend $1^{-/-}$mice; $n=3$ per genotype). Immunofluorescence (I) for the apoptotic marker caspase-3 and quantification in $\mathbf{( J )}$ shows a significant increase in cell death in Cend $1^{-/-}$mice $\left(1.33 \pm 0.33\right.$ in wt vs. $3.66 \pm 0.33$ in Cend1 ${ }^{-1-}$ mice; $n=4$ per genotype). Nuclei (blue) are visualized with TOPRO3 in (D, I). Values represent mean \pm SEM. Scale bars (B, D), $100 \mu \mathrm{m} ;(\mathbf{F}), 50 \mu \mathrm{m} ;{ }^{*} P<0.05$; $* * P<0.01$

PV; 39\%, P = 0.013 for Sst; Figure 8). However, unlike PV interneurons, we did not observe a similar reduction in the $\mathrm{Sst}^{+}$population within the hilus of the DG in Cend1 $1^{-/-}$ mice (Figure 8). This data in conjunction with the aberrant adult hippocampal neurogenesis underscore the contribution of Cend 1 in learning and memory processes. 

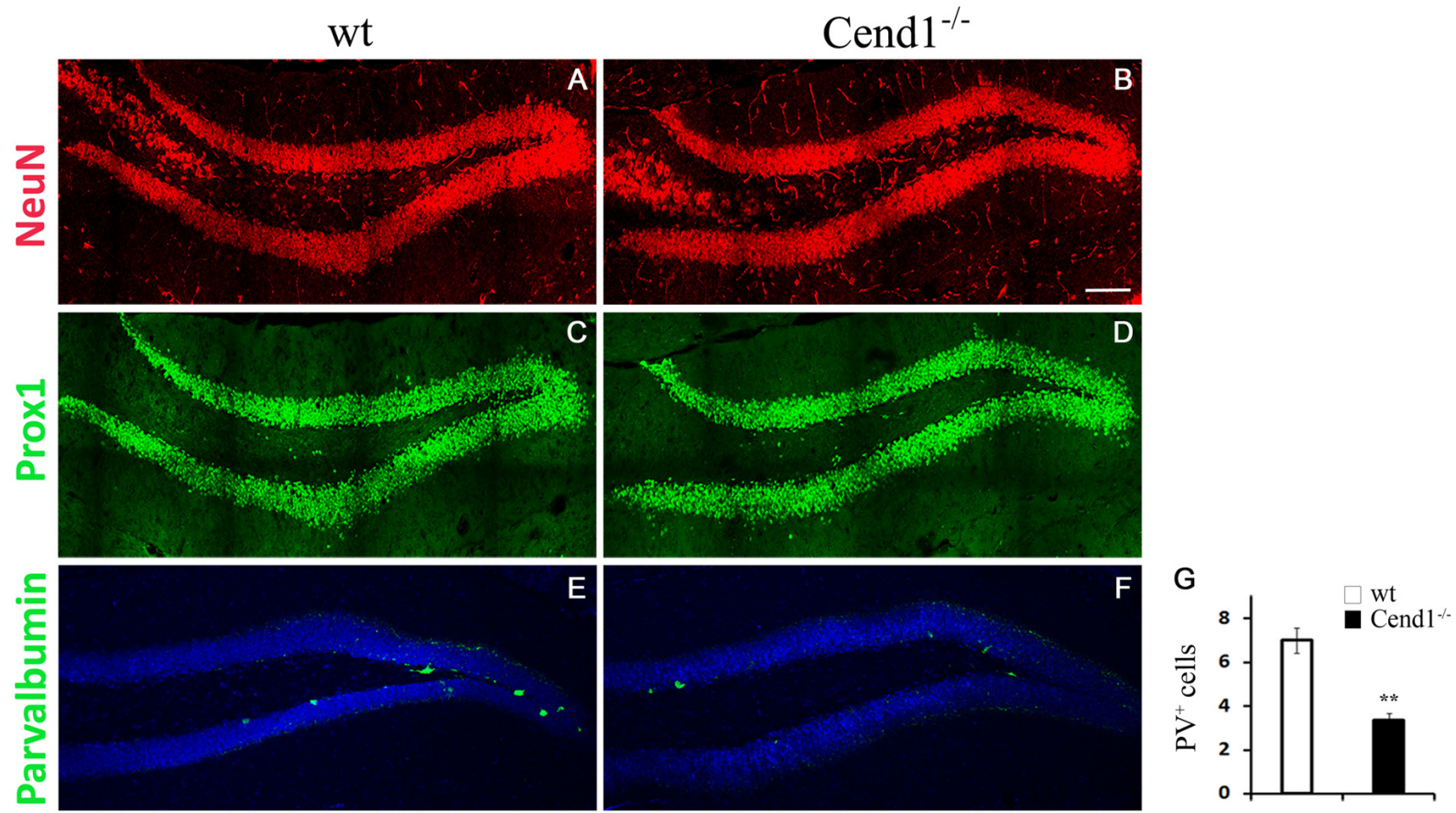

FIGURE 6 | Decreased PV-expressing interneurons in the hippocampal dentate gyrus (DG) of adult Cend1 $1^{-/-}$mice. Cryostat sections immunostained for the mature neuronal marker NeuN (A,B) and the DG granule neuron marker Prox1 (C,D), show similar expression patters between genotypes. Immunofluorescence labeling (E,F) and quantification (G) reveals a significant reduction in PV-expressing interneurons in the DG of Cend1 ${ }^{-/-}$mice $(7.0 \pm 0.5 \mathrm{PV}$ cells in wt vs. $3.33 \pm 0.33$ in Cend $1^{-/-}$mice; $n=3$ mice per genotype). Nuclei are visualized with TOPRO3. Values represent mean \pm SEM. Scale bar (B), $100 \mu$ m; ${ }^{* *} P<0.01$.

\section{DISCUSSION}

In this work we identified increased anxiety-related behavior and cognitive impairment in adult Cend1-deficient mice that were accompanied by cellular alterations in functionally relevant brain areas. These included a decrease in the density of GABAergic interneurons in the somatosensory cortex, the hippocampus and the amygdala, particularly in the basolateral nucleus, and aberrant hippocampal neurogenesis. The decrease in GABAergic interneurons in the cortex and amygdala identified in $\mathrm{Cend}^{-/}$mice was associated with higher proliferation and apoptosis as well as reduced migration of neuronal progenitors from the embryonic GEs where these cells originate. Further, we showed that Cend 1 specifically influences the generation and/or differentiation of GABAergic neurons since glutamatergic cells are not affected, at least in the cortex. These observations complement our previous studies suggesting that Cend1 is a molecular determinant of the neuronal lineage coordinating cell cycle exit and differentiation of neuronal precursors (Koutmani et al., 2004; Georgopoulou et al., 2006; Politis et al., 2007a,b). In support, gain- and lossof-function experiments in neural stem/ progenitor cells have shown that Cend1 prompts neuronal precursors to exit the cell cycle and differentiate to a neuronal fate (Katsimpardi et al., 2008; Makri et al., 2010). Our first report on the characterization of Cend $1^{-/}$mice revealed irregularities in cerebellar lamination that resulted in ataxic gait and deficits in motor coordination (Sergaki et al., 2010). Consistent with Cend 1 function, these defects arose from enhanced proliferation of granule cell precursors, delayed radial migration of granule cells and compromised Purkinje cell differentiation. Collectively, our previous and current data highlight the necessity of Cend1 expression both during development and in the adult for establishment of a structurally and functionally normal phenotype.

GABA and glutamate are the two main neurotransmitters in the adult brain, which sustain the inhibitory-excitatory balance that is essential for proper function (Koós and Tepper, 1999; Markram et al., 2004; Xu et al., 2011; Takesian and Hensch, 2013). Inhibitory networks of GABAergic interneurons in the amygdala have a central role in shaping anxiety responses in the normal brain and at pathological states (Nuss, 2015). A decline in the GABAergic population resulting in reduced inhibitory neurotransmission in the amygdala is consistent with increased levels of anxiety (LeDoux, 2003), with the basolateral and central nuclei especially being involved in this process (Tye et al., 2011). Several lines of evidence indicate that additional brain circuits comprising top-down mechanisms originating in the cortex also modulate anxiety responses. Thus, in the somatosensory cortex, a reduction in local circuit inhibition due to lower levels of GABAergic interneurons was accompanied by increased anxiety (Matsuda et al., 2011). These findings suggest a causal relation between the increased anxiety-related behavior seen in Cend1 $1^{-/-}$mice 

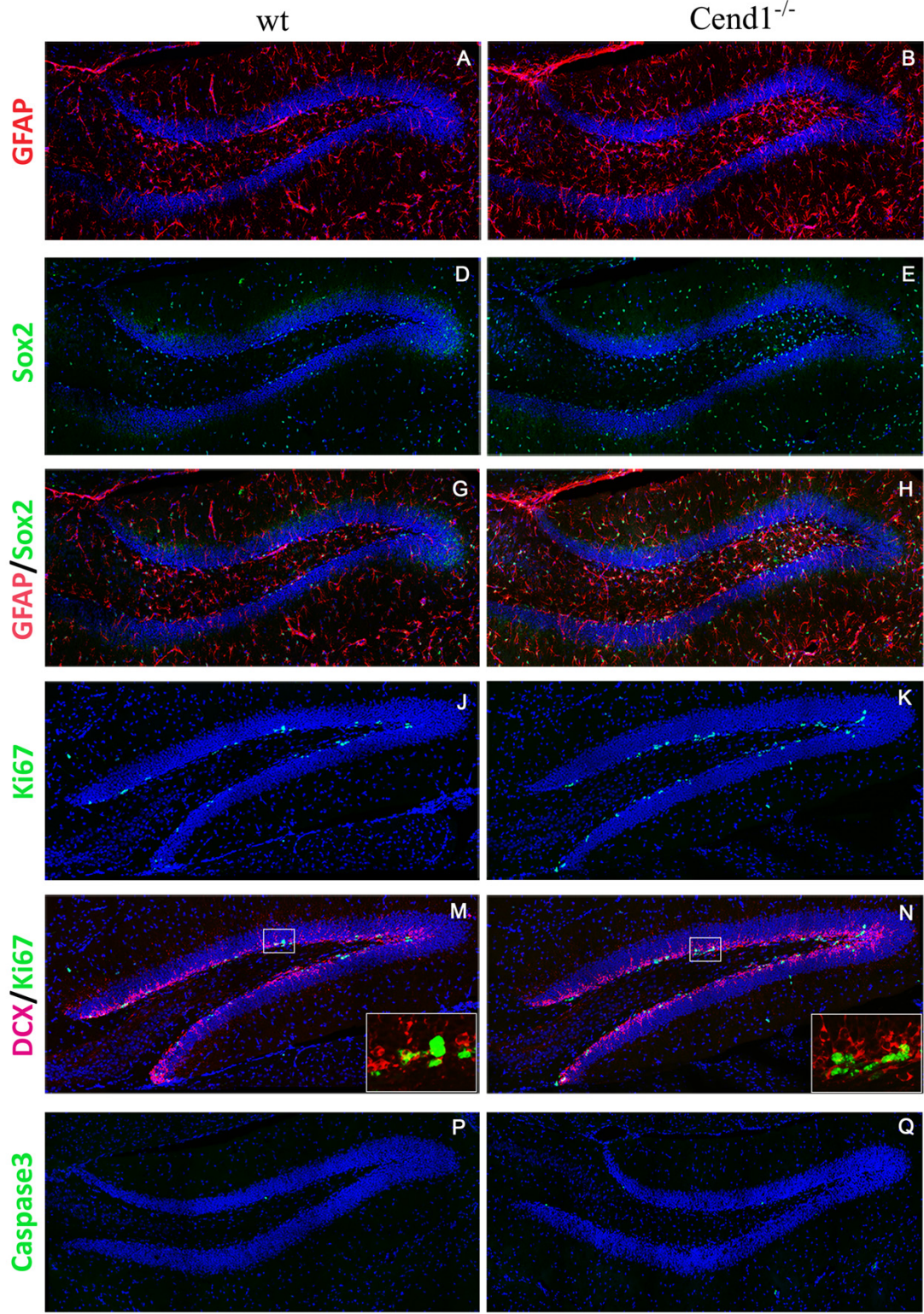
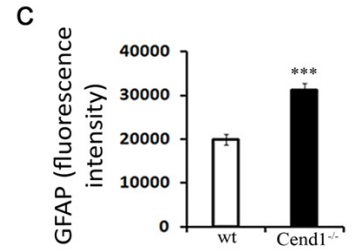

$\mathbf{F}$

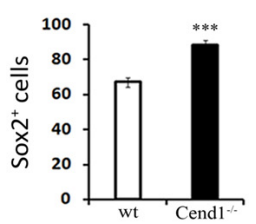

I
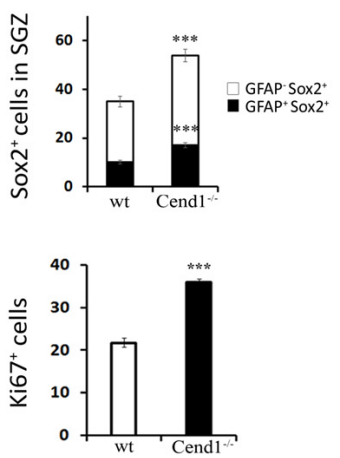

O

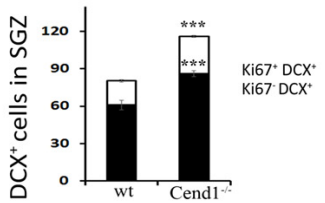

$\mathbf{R}$

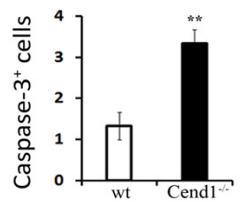

FIGURE 7 | Aberrant neurogenesis in the hippocampus of adult Cend1-/- mice. (A-I) Cryostat sections of the hippocampal DG double-labeled for GFAP (red) and Sox2 (green). Nuclei are seen with TOPRO3 (blue). Quantification of GFAP fluorescence intensity is shown in [(C); $19904 \pm 1242$ arbitrary units in wt vs. $31284 \pm 1502$ in Cend1 $1^{-1-}$ mice; $n=3$ mice per genotype] and of the total number of Sox $2^{+}$cells in $\left[(\mathbf{F}) ; 67.0 \pm 2.7\right.$ vs. $88.3 \pm 2.5$ in Cend1 ${ }^{-1-}$ mice; $n=3$ mice per genotype]. The numbers of GFAP ${ }^{+} / \mathrm{Sox} 2^{+}$neural stem cells and GFAP ${ }^{-} / \mathrm{Sox} 2^{+}$precursor cells in the subgranular zone (SGZ) of the DG are quantified in (I; $10.1 \pm 0.8$ in wt vs. $17.2 \pm 1.0 \mathrm{GFAP}^{+} / \mathrm{Sox}^{+}$cells in Cend $1^{-/-}$mice and $24.83 \pm 2.2$ in wt vs. $36.66 \pm 2.45 \mathrm{GFAP}^{-} /$Sox $^{+}$cells in Cend $1^{-/-}$mice; $n=3$ per genotype). (J,K) Immunofluorescence labeling for the proliferation marker Ki67 and quantification (L) reveals a significant increase of Ki67 ${ }^{+}$cells in the SGZ of Cend $1^{-1-}$ mice $\left(21.8 \pm 0.9 \mathrm{Ki} 67^{+}\right.$cells in wt vs. $36.0 \pm 0.9$ in Cend $1^{-/-}$mice; $n=3$ per genotype). Double immunofluorescence labeling for the neuroblast/newborn neuron marker doublecortin (DCX) and the proliferation marker Ki67 (M,N) and quantification (O) shows an increase in both proliferating neuroblasts and non-proliferating newborn neurons $\left(19.4 \pm 1.1 \mathrm{DCX}+/ \mathrm{Ki}^{6} 7^{+}\right.$cycling neuroblasts in wt vs. $30.0 \pm 0.8$ in Cend1 ${ }^{-/-}$mice; $60.9 \pm 3.9 \mathrm{DCX}+/ \mathrm{Ki}^{-} 7^{-}$newborn neurons in wt vs. $86.16 \pm 2.54$ in Cend $1^{-/-}$mice; $n=3$ per genotype). (P,Q) Immunofluorescence labeling for the apoptotic marker activated caspase-3 and quantification (R), shows a significant increase of cell death in the SGZ of Cend $1^{-1-}$ mice $\left(1.33 \pm 0.33\right.$ caspase- $3^{+}$cells in wt vs. $3.33 \pm 0.33$ in Cend $1^{-/-}$mice; $n=3$ per genotype). Values represent means \pm SEM. Scale bar (Q), $100 \mu \mathrm{m} ;{ }^{* *} P<0.01 ;{ }^{* * *} P<0.005$

and the decrease in GABAergic interneurons observed in both the basolateral nucleus of the amygdala and the somatosensory cortex. Interestingly, not all interneuron subtypes were equally affected in a given brain region. In the cortex of Cend1 $1^{-/-}$ mice both PV and calbindin expressing neurons were reduced as well as Sst-expressing cells, which comprise a much smaller interneuron population. On the other hand in the BLA, only calbindin, but not PV, neurons were different between genotypes. 

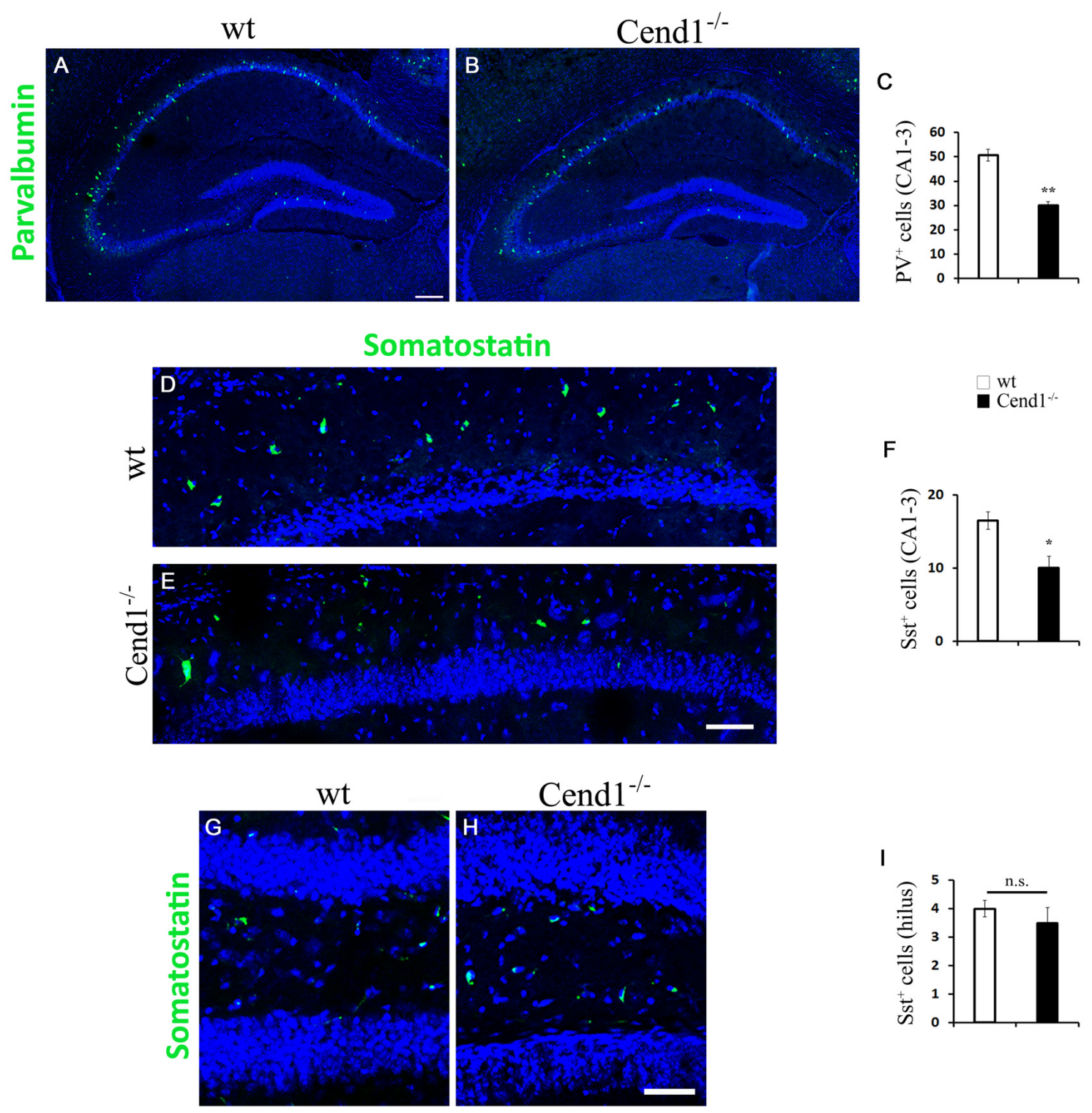

FIGURE 8 | Decreased PV- and Sst-expressing interneurons in the hippocampal CA1-3 regions of adult Cend1-/- mice. (A,B) Cryostat sections of the hippocampus, including the CA1-3 areas, immunostained for PV (green). Quantification (C) reveals a significant decrease of $\mathrm{PV}{ }^{+}$cells in the CA1-3 regions of Cend1 ${ }^{-/-}$mice $\left(50.73 \pm 2.42 \mathrm{PV}^{+}\right.$cells in wt vs. $30 \pm 1.52$ in Cend1 ${ }^{-/-}$mice; $n=3$ per genotype). (D,E) Immunofluorescence labeling for Sst (green) in CA1 and quantification in CA1-3 (F), shows a significant decrease in this interneuron subtype in Cend1 ${ }^{-/-}$mice $\left(16.5 \pm 1.19\right.$ Sst ${ }^{+}$cells in wt vs. $10 \pm 1.58$ in Cend1 ${ }^{-/-}$

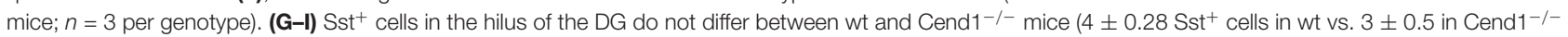
mice; $n=3$ per genotype). Nuclei are seen with TOPRO3 (blue). Values represent means \pm SEM. Scale bar (A,E), $200 \mu$ m; (H), $50 \mu m$; ${ }^{*} P<0.05,{ }^{* *} P<0.01 ; \mathrm{n} . \mathrm{s}$, non significant.

Interestingly, while $\mathrm{PV}^{+}$BLA neurons have been shown not to be implicated in emotional memory (Burghardt et al., 2006; Bienvenu et al., 2012; Butler et al., 2017), calbindin ${ }^{+}$BLA neurons have been involved in processes underlying synaptic plasticity-mediated encoding of fear memory (Bienvenu et al., 2012).

Cend $1^{-/-}$mice, although exhibiting increased innate fear/anxiety, show reduced freezing following cued fear conditioning. This counter-intuitive behavior could be attributed to a malfunctioning feed-in circuit from sensory cortices to the amygdala. During cued fear conditioning, both the auditory and somatosensory cortices are activated and their proper function is GABA-tuned (Scaife et al., 2007). In order for the aversive association to be formed between the tone (conditioning stimulus-cue) and the noxious stimulus (unconditioned stimulus-pain caused by the electroshock), the two cortices (auditory and somatosensory) have to function efficiently and send convergent and precisely timed information to the 
BLA. If the timing and/or strength of incoming information are insufficient, the association fails to be formed and this is manifested as reduced freezing in future exposures to the conditioning stimulus. It should be noted that a similar behavioral profile with increased innate fear/anxiety and reduced learned (conditioned) fear has been observed in a transgenic mouse overproducing corticotropin-releasing hormone (van Gaalen et al., 2002).

Cend $1^{-/-}$mice display spatial learning and memory defects as determined in the MWM test. To exclude the possibility that this deficit was not associated with cognition but rather with their ataxic phenotype, we checked and confirmed that Cend $1^{-/-}$animals swam normally. This is not surprising, as in other models of ataxic mice, ataxia did not affect the swimming speed of the animals, possibly due to the nature of this measurement: gait-impaired animals can, apparently, compensate in the water with other body movements, or extra hind-limb kicks (Lalonde and Botez, 1986; Savvaki et al., 2008; Jayabal et al., 2015). The cellular changes in the somatosensory cortex and the hippocampus of Cend $1^{-/-}$mice should also contribute to the spatial learning and memory defects noted in these animals; while it is the hippocampus that subserves acquisition and recall of new spatial information, memory storage is eventually mediated by the cortex, with modulation of gene expression in various areas, including the somatosensory, but not the motor, cortex (Park et al., 2011). Particularly regarding the role of $\mathrm{PV}$-positive GABAergic interneurons, it has been shown that a selective inactivation of this sub-population in the cortex affects negatively only cognitive abilities, suggesting that these interneurons may contribute to specific functions of cortical circuits (Murray et al., 2015). Such a divided role between different interneuron populations is further supported by studies showing that targeting of the Sst-positive population of interneurons in the prefrontal cortex of Sst-Cre mice, had no effect on working memory in a task afflicted by the inactivation of PV-expressing interneurons (Taniguchi et al., 2011).

Cend $1^{-/}$mice also display aberrations in hippocampal neurogenesis, which is particularly interesting because of its association with higher cognitive functions, particularly memory processes. In agreement with Cend1 function in other brain areas, we noted a cell proliferation increase in the DG SGZ in Cend $1^{-/-}$mice, resulting in higher numbers of neural stem and progenitor cells as well as in increased numbers of cycling neuroblasts and newborn neurons. These features were paralleled by an increase in local astrocytes, which are known to stimulate neurogenesis (Song et al., 2002), and a decrease in local PV-expressing interneurons. It has been previously reported that $\mathrm{PV}$-expressing interneurons provide immature GABAergic synaptic inputs to proliferating adult mouse hippocampal neural precursors from. Through this mechanism, PV cells suppress the activation of quiescent neural stem cells (Song et al., 2012) while at later stages of neurogenesis they promote survival of neuronal progeny (Song et al., 2013). Therefore, the enhancement of the earlier stages of neurogenesis in Cend $1^{-/-}$mice may be attributed to cell intrinsic mechanisms and/or to cell extrinsic influences arising from the dual effects of an increase in local astrocytes and a decrease in PV interneurons. At the same time, increased cell elimination by apoptotic death may be due to insufficient support provided by the reduced numbers of PV interneurons.

These combined effects might affect the maintenance of a normal neuronal circuitry in the adult hippocampus, contributing to the cognitive impairment of Cend $1^{-/-}$ mice. Apart from their regulatory role in neurogenesis, $\mathrm{PV}^{+}$ GABAergic neurons innervate the cell body of CA1 pyramidal neurons and control their glutamatergic output (Klausberger and Somogyi, 2008). It has been shown that selective removal of PV-expressing neurons leads to impaired spatial memory (Murray et al., 2011), and therefore the decrease in $\mathrm{PV}^{+}$neurons may disturb the overall output, leading to memory defects in Cend $1^{-/-}$mice.

The population of hippocampal Sst neurons shows an area-dependent effect in Cend1-/- mice, being decreased in CA1-3 areas while not being affected in the DG. This difference could be attributed to differences in their developmental profile, as it has been shown that Sst neurons in CA1-3 are born before those in the DG (Rapp and Amaral, 1988). The differential effect of Cend1 ablation on PV and Sst DG neurons is interesting given the distinct role of these inhibitory interneurons in the flow of input from cortical areas to other hippocampal areas, with PV neurons regulating the spiking of DG granule cells while both populations are involved in containing granule cell activity (Lee et al., 2016). Nevertheless, a precise tuning of the functions of both types of interneurons is necessary for proper DG function e.g., during memory formation for filtering out irrelevant information. In contrast to Sst interneurons, the PV neuronal population was decreased throughout the hippocampus, a phenomenon that could also be related to developmental differences of these two types of interneurons; for instance, Sst neurons are produced during the first half of neurogenesis, while PV neurons show a protracted period of birth throughout the neurogenic period (Inan et al., 2012).

In conclusion, the behavioral and cellular alterations we show in the present study elucidate previously unknown aspects of Cend1 involvement during development and in the adult and together with previous data underline the importance of Cend 1 expression for the establishment of a structurally and functionally normal phenotype.

\section{AUTHOR CONTRIBUTIONS}

AS, AL, FS and RM conceived the study. KS, AS and AL designed and performed the experiments; analyzed and interpreted data with the contribution of FS and RM. KS, AS and RM wrote the article with the contribution of FS and AL. RM and FS provided financial support. All authors approved the final manuscript.

\section{FUNDING}

This work was supported by the project "Infectious, autoimmune and neurodegenerative diseases: study of the pathogenetic 
mechanisms and development of diagnostic, prognostic and therapeutic approaches" (MIS 5002486) which is implemented under the "Action for Strategic Development of the Research and Technological Sector", funded by the Operational Program "Competitiveness, Entrepreneurship and Innovation" (NSRF 2014-2020) and co-financed by Greece (Ministry of National Education and Religious Affairs) and the European Union (European Regional Development Fund).

\section{ACKNOWLEDGMENTS}

We would like to thank Dr. Paraskevi Koutsoudaki for excellent assistance in the behavioral experiments, as well as the members of the HPI Light Microscopy Unit for their help using the Imaris image analysis software.

\section{REFERENCES}

Abrous, D. N., and Wojtowicz, J. M. (2015). Interaction between neurogenesis and hippocampal memory system: new vistas. Cold Spring Harb. Perspect. Biol. 7:a018952. doi: 10.1101/cshperspect.a018952

Achim, K., Salminen, M., and Partanen, J. (2014). Mechanisms regulating GABAergic neuron development. Cell. Mol. Life Sci. 71, 1395-1415. doi: 10.1007/s00018-013-1501-3

Arlotta, P., Molyneaux, B. J., Jabaudon, D., Yoshida, Y., and Macklis, J. D. (2008). Ctip2 controls the differentiation of medium spiny neurons and the establishment of the cellular architecture of the striatum. J. Neurosci. 28, 622-632. doi: 10.1523/JNEUROSCI.2986-07.2008

Bertrand, N., Castro, D. S., and Guillemot, F. (2002). Proneural genes and the specification of neural cell types. Nat. Rev. Neurosci. 3, 517-530. doi: $10.1038 / \mathrm{nrn} 874$

Bienvenu, T. C., Busti, D., Magill, P. J., Ferraguti, F., and Capogna, M. (2012). Cell-type-specific recruitment of amygdala interneurons to hippocampal theta rhythm and noxious stimuli in vivo. Neuron 74, 1059-1074. doi: 10.1016/j. neuron.2012.04.022

Burghardt, P. R., Pasumarthi, R. K., Wilson, M. A., and Fadel, J. (2006). Alterations in fear conditioning and amygdalar activation following chronic wheel running in rats. Pharmacol. Biochem. Behav. 84, 306-312. doi: 10.1016/j.pbb.2006. 05.015

Butler, R. K., Ehling, S., Barbar, M., Thomas, J., Hughes, M. A., Smith, C. E., et al. (2017). Distinct neuronal populations in the basolateral and central amygdala are activated with acute pain, conditioned fear, and fear-conditioned analgesia. Neurosci. Lett. 661, 11-17. doi: 10.1016/j.neulet.2017. 09.025

Castro, D. S., Martynoga, B., Parras, C., Ramesh, V., Pacary, E., Johnston, C., et al. (2011). A novel function of the proneural factor Ascll in progenitor proliferation identified by genome-wide characterization of its targets. Genes Dev. 25, 930-945. doi: 10.1101/gad.627811

Catavero, C., Bao, H., and Song, J. (2018). Neural mechanisms underlying GABAergic regulation of adult hippocampal neurogenesis. Cell Tissue Res. 371, 33-46. doi: 10.1007/s00441-017-2668-y

Cheng, L., Arata, A., Mizuguchi, R., Qian, Y., Karunaratne, A., Gray, P. A., et al. (2004). Tlx3 and Tlx1 are post-mitotic selector genes determining glutamatergic over GABAergic cell fates. Nat. Neurosci. 7, 510-517. doi: $10.1038 / \mathrm{nn} 1221$

Crair, M. C. (1999). Neuronal activity during development: permissive or instructive? Curr. Opin. Neurobiol. 9, 88-93. doi: 10.1016/s0959-4388(99) 80011-7

D'Hooge, R., and De Deyn, P. P. (2001). Applications of the Morris water maze in the study of learning and memory. Brain Res. Rev. 36, 60-90. doi: 10.1016/s0165-0173(01)00067-4

Ehrlich, I., Humeau, Y., Grenier, F., Ciocchi, S., Herry, C., and Lüthi, A. (2009). Amygdala inhibitory circuits and the control of fear memory. Neuron 62, 757-771. doi: 10.1016/j.neuron.2009.05.026

\section{SUPPLEMENTARY MATERIAL}

The Supplementary Material for this article can be found online at: https://www.frontiersin.org/articles/10.3389/fncel. 2018.00497/full\#supplementary-material

FIGURE S1 | No difference in the density of glutamatergic projection neurons in the adult cortex of Cend1 $1^{-1-}$ mice. Confocal images of cryostat sections of adult somatosensory cortex immunostained for Satb2 (A) and quantification of Satb2 ${ }^{+}$ cells in Cend1 $1^{-/-}$mice as compared to wt animals (B). Values represent mean \pm SEM. Scale bar (A), $100 \mu \mathrm{m}$.

FIGURE S2 | No evidence for inflammatory microgliosis. Double labeling for the microglial/macrophage marker lba1 (red) and the activated microglial/macrophage marker CD68 (green) shows no signs of inflammatory microgliosis in the hippocampal DG of Cend1-/- mice as compared with wt mice. Nuclei are counterstained with TOPRO3. Scale bar $100 \mu \mathrm{m}$.

Eriksson, P. S., Perfilieva, E., Björk-Eriksson, T., Alborn, A. M., Nordborg, C., Peterson, D. A., et al. (1998). Neurogenesis in the adult human hippocampus. Nat. Med. 4, 1313-1317. doi: 10.1038/3305

Georgopoulou, N., Hurel, C., Politis, P. K., Gaitanou, M., Matsas, R., and Thomaidou, D. (2006). BM88 is a dual function molecule inducing cell cycle exit and neuronal differentiation of neuroblastoma cells via cyclin D1 down-regulation and retinoblastoma protein hypophosphorylation. J. Biol. Chem. 281, 33606-33620. doi: 10.1074/jbc.M602689200

Götz, M., and Huttner, W. B. (2005). The cell biology of neurogenesis. Nat. Rev. Mol. Cell Biol. 6, 777-788. doi: 10.1038/nrm1739

Götz, M., Nakafuku, M., and Petrik, D. (2016). Neurogenesis in the developing and adult brain-similarities and key differences. Cold Spring Harb. Perspect. Biol. 8:a018853. doi: 10.1101/cshperspect.a018853

Hardwick, L. J., Ali, F. R., Azzarelli, R., and Philpott, A. (2015). Cell cycle regulation of proliferation versus differentiation in the central nervous system. Cell Tissue Res. 359, 187-200. doi: 10.1007/s00441-014-1895-8

Inan, M., Welagen, J., and Anderson, S. A. (2012). Spatial and temporal bias in the mitotic origins of somatostatin- and parvalbumin-expressing interneuron subgroups and the chandelier subtype in the medial ganglionic eminence. Cereb. Cortex 22, 820-827. doi: 10.1093/cercor/bhr148

Jayabal, S., Ljungberg, L., Erwes, T., Cormier, A., Quilez, S., El Jaouhari, S., et al. (2015). Rapid onset of motor deficits in a mouse model of spinocerebellar ataxia type 6 precedes late cerebellar degeneration. eNeuro 2:ENEURO.0094-15.2015. doi: 10.1523/eneuro.0094-15.2015

Jessell, T. M. (2000). Neuronal specification in the spinal cord: inductive signals and transcriptional codes. Nat. Rev. Genet. 1, 20-29. doi: 10.1038/350 49541

Katsimpardi, L., Gaitanou, M., Malnou, C. E., Lledo, P. M., Charneau, P., Matsas, R., et al. (2008). BM88/Cend1 expression levels are critical for proliferation and differentiation of subventricular zone-derived neural precursor cells. Stem Cells 26, 1796-1807. doi: 10.1634/stemcells.2007-0921

Kee, N., Teixeira, C. M., Wang, A. H., and Frankland, P. W. (2007). Preferential incorporation of adult-generated granule cells into spatial memory networks in the dentate gyrus. Nat. Neurosci. 10, 355-362. doi: 10.1038/nn1847

Kelsom, C., and Lu, W. (2013). Development and specification of GABAergic cortical interneurons. Cell Biosci. 3:19. doi: 10.1186/2045-3701-3-19

Kempermann, G., Jessberger, S., Steiner, B., and Kronenberg, G. (2004). Milestones of neuronal development in the adult hippocampus. Trends Neurosci. 27, 447-452. doi: 10.1016/j.tins.2004.05.013

Kempermann, G., Song, H., and Gage, F. H. (2015). Neurogenesis in the adult hippocampus. Cold Spring Harb. Perspect. Biol. 7:a018812. doi: $10.1101 /$ cshperspect.a018812

Klausberger, T., and Somogyi, P. (2008). Neuronal diversity and temporal dynamics: the unity of hippocampal circuit operations. Science 321, 53-57. doi: $10.1126 /$ science. 1149381

Koós, T., and Tepper, J. M. (1999). Inhibitory control of neostriatal projection neurons by GABAergic interneurons. Nat. Neurosci. 2, 467-472. doi: $10.1038 / 8138$ 
Kouroupi, G., Lavdas, A. A., Gaitanou, M., Thomaidou, D., Stylianopoulou, F., and Matsas, R. (2010). Lentivirus-mediated expression of insulin-like growth factor-I promotes neural stem/precursor cell proliferation and enhances their potential to generate neurons. J. Neurochem. 115, 460-474. doi: 10.1111/j.14714159.2010.06939.x

Koutmani, Y., Hurel, C., Patsavoudi, E., Hack, M., Gotz, M., Thomaidou, D., et al. (2004). BM88 is an early marker of proliferating precursor cells that will differentiate into the neuronal lineage. Eur. J. Neurosci. 20, 2509-2523. doi: 10.1111/j.1460-9568.2004.03724.x

Lalonde, R., and Botez, M. I. (1986). Navigational deficits in weaver mutant mice. Brain Res. 398, 175-177. doi: 10.1016/0006-8993(86)91264-3

Lavdas, A. A., Grigoriou, M., Pachnis, V., and Parnavelas, J. G. (1999). The medial ganglionic eminence gives rise to a population of early neurons in the developing cerebral cortex. J. Neurosci. 19, 7881-7888. doi: 10.1523/JNEUROSCI.19-18-07881.1999

Le Magueresse, C., and Monyer, H. (2013). GABAergic interneurons shape the functional maturation of the cortex. Neuron 77, 388-405. doi: 10.1016/j.neuron. 2013.01.011

LeDoux, J. E. (2000). Emotion circuits in the brain. Annu. Rev. Neurosci. 23, 155-184. doi: 10.1146/annurev.neuro.23.1.155

LeDoux, J. (2003). The emotional brain, fear, and the amygdala. Cell. Mol. Neurobiol. 23, 727-738. doi: 10.1023/A:1025048802629

Lee, C. T., Kao, M. H., Hou, W. H., Wei, Y. T., Chen, C. L., and Lien, C. C. (2016). Causal evidence for the role of specific GABAergic interneuron types in entorhinal recruitment of dentate granule cells. Sci. Rep. 6:36885. doi: $10.1038 /$ srep36885

Lehmann, K., Steinecke, A., and Bolz, J. (2012). GABA through the ages: regulation of cortical function and plasticity by inhibitory interneurons. Neural Plast. 2012:892784. doi: 10.1155/2012/892784

Leuner, B., Mendolia-Loffredo, S., Kozorovitskiy, Y., Samburg, D., Gould, E., and Shors, T. J. (2004). Learning enhances the survival of new neurons beyond the time when the hippocampus is required for memory. J. Neurosci. 24, 7477-7481. doi: 10.1523/JNEUROSCI.0204-04.2004

Löw, K., Crestani, F., Keist, R., Benke, D., Brünig, I., Benson, J. A., et al. (2000). Molecular and neuronal substrate for the selective attenuation of anxiety. Science 290, 131-134. doi: 10.1126/science.290.5489.131

Makri, G., Lavdas, A. A., Katsimpardi, L., Charneau, P., Thomaidou, D., and Matsas, R. (2010). Transplantation of embryonic neural stem/precursor cells overexpressing BM88/Cend1 enhances the generation of neuronal cells in the injured mouse cortex. Stem Cells 28, 127-139. doi: 10.1002/ stem. 258

Marín, O., and Rubenstein, J. L. (2003). Cell migration in the forebrain. Annu. Rev. Neurosci. 26, 441-483. doi: 10.1146/annurev.neuro.26.041002.131058

Markram, H., Toledo-Rodriguez, M., Wang, Y., Gupta, A., Silberberg, G., and $\mathrm{Wu}, \mathrm{C}$. (2004). Interneurons of the neocortical inhibitory system. Nat. Rev. Neurosci. 5, 793-807. doi: 10.1038/nrn1519

Marquardt, T., and Pfaff, S. L. (2001). Cracking the transcriptional code for cell specification in the neural tube. Cell 106, 651-654. doi: 10.1016/s00928674(01)00499-8

Matsuda, Y., Inoue, Y., Izumi, H., Kaga, M., Inagaki, M., and Goto, Y. (2011). Fewer GABAergic interneurons, heightened anxiety and decreased high-frequency electroencephalogram components in Bronx waltzer mice, a model of hereditary deafness. Brain Res. 1373, 202-210. doi: 10.1016/j.brainres. 2010.12.006

Murray, A. J., Sauer, J. F., Riedel, G., McClure, C., Ansel, L., Cheyne, L., et al. (2011). Parvalbumin-positive CA1 interneurons are required for spatial working but not for reference memory. Nat. Neurosci. 14, 297-299. doi: $10.1038 / \mathrm{nn} .2751$

Murray, A. J., Woloszynowska-Fraser, M. U., Ansel-Bollepalli, L., Cole, K. L., Foggetti, A., Crouch, B., et al. (2015). Parvalbumin-positive interneurons of the prefrontal cortex support working memory and cognitive flexibility. Sci. Rep. 5:16778. doi: 10.1038/srep16778

Nakajima, K. (2007). Control of tangential/non-radial migration of neurons in the developing cerebral cortex. Neurochem. Int. 51, 121-131. doi: 10.1016/j.neuint. 2007.05.006

Nery, S., Fishell, G., and Corbin, J. G. (2002). The caudal ganglionic eminence is a source of distinct cortical and subcortical cell populations. Nat. Neurosci. 5, 1279-1287. doi: 10.1038/nn971
Nicola, Z., Fabel, K., and Kempermann, G. (2015). Development of the adult neurogenic niche in the hippocampus of mice. Front. Neuroanat. 9:53. doi: 10.3389 /fnana.2015.00053

Nuss, P. (2015). Anxiety disorders and GABA neurotransmission: a disturbance of modulation. Neuropsychiatr. Dis. Treat. 11, 165-175. doi: 10.2147/ndt. s58841

Ognjanovski, N., Schaeffer, S., Wu, J., Mofakham, S., Maruyama, D., Zochowski, M., et al. (2017). Parvalbumin-expressing interneurons coordinate hippocampal network dynamics required for memory consolidation. Nat. Commun. 8:15039. doi: 10.1038/ncomms15039

Ohnuma, S., and Harris, W. A. (2003). Neurogenesis and the cell cycle. Neuron 40, 199-208. doi: 10.1016/S0896-6273(03)00632-9

Papastefanaki, F., Jakovcevski, I., Poulia, N., Djogo, N., Schulz, F., Martinovic, T., et al. (2015). Intraspinal delivery of polyethylene glycol-coated gold nanoparticles promotes functional recovery after spinal cord injury. Mol. Ther. 23, 993-1002. doi: 10.1038/mt.2015.50

Parent, J. M. (2007). Adult neurogenesis in the intact and epileptic dentate gyrus. Prog. Brain Res. 163, 529-540. doi: 10.1016/s0079-6123(07)63028-3

Park, S. S., Stranahan, A. M., Chadwick, W., Zhou, Y., Wang, L., Martin, B., et al. (2011). Cortical gene transcription response patterns to water maze training in aged mice. BMC Neurosci. 12:63. doi: 10.1186/1471-2202-12-63

Politis, P. K., Makri, G., Thomaidou, D., Geissen, M., Rohrer, H., and Matsas, R. (2007a). BM88/CEND1 coordinates cell cycle exit and differentiation of neuronal precursors. Proc. Natl. Acad. Sci. U S A 104, 17861-17866. doi: 10.1073/pnas.0610973104

Politis, P. K., Rohrer, H., and Matsas, R. (2007b). Expression pattern of BM88 in the developing nervous system of the chick and mouse embryo. Gene Expr. Patterns 7, 165-177. doi: 10.1016/j.modgep.2006.06.007

Qian, X., Shen, Q., Goderie, S. K., He, W., Capela, A., Davis, A. A., et al. (2000). Timing of CNS cell generation: a programmed sequence of neuron and glial cell production from isolated murine cortical stem cells. Neuron 28, 69-80. doi: 10.1016/S0896-6273(00)00086-6

Ramirez-Amaya, V., Marrone, D. F., Gage, F. H., Worley, P. F., and Barnes, C. A. (2006). Integration of new neurons into functional neural networks. J. Neurosci. 26, 12237-12241. doi: 10.1523/JNEUROSCI.2195-06.2006

Rapp, P. R., and Amaral, D. G. (1988). The time of origin of somatostatinimmunoreactive neurons in the rat hippocampal formation. Dev. Brain Res. 469, 231-239. doi: 10.1016/0165-3806(88)90185-x

Roozendaal, B., McEwen, B. S., and Chattarji, S. (2009). Stress, memory and the amygdala. Nat. Rev. Neurosci. 10, 423-433. doi: 10.1038/nrn2651

Saleem, A. B., Ayaz, A., Jeffery, K. J., Harris, K. D., and Carandini, M. (2013). Integration of visual motion and locomotion in mouse visual cortex. Nat. Neurosci. 16, 1864-1869. doi: 10.1038/nn.3567

Savvaki, M., Panagiotaropoulos, T., Stamatakis, A., Sargiannidou, I., Karatzioula, P., Watanabe, K., et al. (2008). Impairment of learning and memory in TAG-1 deficient mice associated with shorter CNS internodes and disrupted juxtaparanodes. Mol. Cell. Neurosci. 39, 478-490. doi: 10.1016/j.mcn. 2008.07.025

Scaife, J. C., Hou, R. H., Samuels, E. R., Baqui, F., Langley, R. W., Bradshaw, C. M., et al. (2007). Diazepam-induced disruption of classicallyconditioned fear-potentiation of late-latency auditory evoked potentials is prevented by flumazenil given before, but not after, CS/US pairing. J. Psychopharmacol. 21, 93-101. doi: 10.1177/0269881106063130

Sergaki, M. C., Guillemot, F., and Matsas, R. (2010). Impaired cerebellar development and deficits in motor coordination in mice lacking the neuronal protein BM88/Cend1. Mol. Cell. Neurosci. 44, 15-29. doi: 10.1016/j.mcn.2010. 01.011

Shors, T. J., Miesegaes, G., Beylin, A., Zhao, M., Rydel, T., and Gould, E. (2001). Neurogenesis in the adult is involved in the formation of trace memories. Nature 410, 372-376. doi: 10.1038/414938a

Snyder, J. S., Hong, N. S., McDonald, R. J., and Wojtowicz, J. M. (2005). A role for adult neurogenesis in spatial long-term memory. Neuroscience 130, 843-852. doi: 10.1016/j.neuroscience.2004.10.009

Song, H., Stevens, C. F., and Gage, F. H. (2002). Astroglia induce neurogenesis from adult neural stem cells. Nature 417, 39-44. doi: 10.1038/417039a

Song, J., Sun, J., Moss, J., Wen, Z., Sun, G. J., Hsu, D., et al. (2013). Parvalbumin interneurons mediate neuronal circuitry-neurogenesis coupling in the adult hippocampus. Nat. Neurosci. 16, 1728-1730. doi: 10.1038/nn.3572 
Song, J., Zhong, C., Bonaguidi, M. A., Sun, G. J., Hsu, D., Gu, Y., et al. (2012). Neuronal circuitry mechanism regulating adult quiescent neural stem-cell fate decision. Nature 489, 150-154. doi: 10.1038/nature11306

Takesian, A. E., and Hensch, T. K. (2013). Balancing plasticity/stability across brain development. Prog. Brain Res. 207, 3-34. doi: 10.1016/b978-0-444-633279.00001-1

Taniguchi, H., He, M., Wu, P., Kim, S., Paik, R., Sugino, K., et al. (2011). A resource of Cre driver lines for genetic targeting of GABAergic neurons in cerebral cortex. Neuron 71, 995-1013. doi: 10.1016/j.neuron.2011.07.026

Tashiro, A., Makino, H., and Gage, F. H. (2007). Experience-specific functional modification of the dentate gyrus through adult neurogenesis: a critical period during an immature stage. J. Neurosci. 27, 3252-3259. doi: 10.1523/JNEUROSCI.4941-06.2007

Terzenidou, M. E., Segklia, A., Kano, T., Papastefanaki, F., Karakostas, A., Charalambous, M., et al. (2017). Novel insights into SLC25A46-related pathologies in a genetic mouse model. PLoS Genet. 13:e1006656. doi: 10.1371/journal.pgen.1006656

Trouche, S., Bontempi, B., Roullet, P., and Rampon, C. (2009). Recruitment of adult-generated neurons into functional hippocampal networks contributes to updating and strengthening of spatial memory. Proc. Natl. Acad. Sci. U S A 106, 5919-5924. doi: 10.1073/pnas.0811054106

Tye, K. M., Prakash, R., Kim, S. Y., Fenno, L. E., Grosenick, L., Zarabi, H., et al. (2011). Amygdala circuitry mediating reversible and bidirectional control of anxiety. Nature 471, 358-362. doi: 10.1038/nature09820

van Gaalen, M. M., Stenzel-Poore, M. P., Holsboer, F., and Steckler, T. (2002). Effects of transgenic overproduction of $\mathrm{CRH}$ on anxiety-like behaviour. Eur. J. Neurosci. 15, 2007-2015. doi: 10.1046/j.1460-9568.2002.02040.x

Vanderhaeghen, P., and Polleux, F. (2004). Developmental mechanisms patterning thalamocortical projections: intrinsic, extrinsic and in between. Trends Neurosci. 27, 384-391. doi: 10.1016/j.tins.2004.05.009
Vidaki, M., Tivodar, S., Doulgeraki, K., Tybulewicz, V., Kessaris, N., Pachnis, V., et al. (2012). Racl-dependent cell cycle exit of MGE precursors and GABAergic interneuron migration to the cortex. Cereb. Cortex 22, 680-692. doi: 10.1093/cercor/bhr145

Waclaw, R. R., Ehrman, L. A., Pierani, A., and Campbell, K. (2010). Developmental origin of the neuronal subtypes that comprise the amygdalar fear circuit in the mouse. J. Neurosci. 30, 6944-6953. doi: 10.1523/JNEUROSCI.5772-09.2010

Winocur, G., Wojtowicz, J. M., Sekeres, M., Snyder, J. S., and Wang, S. (2006). Inhibition of neurogenesis interferes with hippocampus-dependent memory function. Hippocampus 16, 296-304. doi: 10.1002/hipo.20163

Xu, G., Broadbelt, K. G., Haynes, R. L., Folkerth, R. D., Borenstein, N. S., Belliveau, R. A., et al. (2011). Late development of the GABAergic system in the human cerebral cortex and white matter. J. Neuropathol. Exp. Neurol. 70, 841-858. doi: 10.1097/nen.0b013e31822f471c

Xu, J. C., Xiao, M. F., Jakovcevski, I., Sivukhina, E., Hargus, G., Cui, Y. F., et al. (2014). The extracellular matrix glycoprotein tenascin-R regulates neurogenesis during development and in the adult dentate gyrus of mice. J. Cell Sci. 127, 641-652. doi: 10.1242/jcs.137612

Conflict of Interest Statement: The authors declare that the research was conducted in the absence of any commercial or financial relationships that could be construed as a potential conflict of interest.

Copyright (C) 2019 Segklia, Stamatakis, Stylianopoulou, Lavdas and Matsas. This is an open-access article distributed under the terms of the Creative Commons Attribution License (CC BY). The use, distribution or reproduction in other forums is permitted, provided the original author(s) and the copyright owner(s) are credited and that the original publication in this journal is cited, in accordance with accepted academic practice. No use, distribution or reproduction is permitted which does not comply with these terms. 